Draft VERSion MARCh 22, 2022

Preprint typeset using $\mathrm{LAT}_{\mathrm{E}} \mathrm{X}$ style emulateapj v. 01/23/15

\title{
A THEORETICAL STUDY OF THE BUILD-UP OF THE SUN'S POLAR MAGNETIC FIELD BY USING A 3D KINEMATIC DYNAMO MODEL
}

\author{
Gopal Hazra $^{1,2}$, Arnab Rai Choudhuri ${ }^{1}$ and Mark S. Miesch ${ }^{3}$ \\ ${ }^{1}$ Department of Physics, Indian Institute of Science, Bangalore, India 560012 \\ ${ }^{2}$ Indian Institute of Astrophysics, Bangalore, India 560034 and \\ ${ }^{3}$ High Altitude Observatory, National Center for Atmospheric Research, Boulder, CO 80301, USA \\ Draft version March 22, 2022
}

\begin{abstract}
We develop a three-dimensional kinematic self-sustaining model of the solar dynamo in which the poloidal field generation is from tilted bipolar sunspot pairs placed on the solar surface above regions of strong toroidal field by using the SpotMaker algorithm, and then the transport of this poloidal field to the tachocline is primarily caused by turbulent diffusion. We obtain a dipolar solution within a certain range of parameters. We use this model to study the build-up of the polar magnetic field and show that some insights obtained from surface flux transport (SFT) models have to be revised. We present results obtained by putting a single bipolar sunspot pair in a hemisphere and two symmetrical sunspot pairs in two hemispheres. We find that the polar fields produced by them disappear due to the upward advection of poloidal flux at low latitudes, which emerges as oppositely-signed radial flux and which is then advected poleward by the meridional flow. We also study the effect that a large sunspot pair, violating Hale's polarity law would have on the polar field. We find that there would be some effect - especially if the anti-Hale pair appears at high latitudes in the mid-phase of the cycle - though the effect is not very dramatic.
\end{abstract}

\section{INTRODUCTION}

The flux transport dynamo model, which started being developed from the 1990s (Wang et al. 1991; Choudhuri et al. 1995; Durney 1995), has emerged as an attractive theoretical model for explaining the solar cycle and has been extensively reviewed by several authors (Choudhuri 2011; Charbonneau 2014; Karak et al. 2014). In any dynamo model, the toroidal magnetic field is generated from the poloidal field by the differential rotation, which has now been mapped by helioseismology (Thompson et al. 1996). The distinctive features of the B-L flux transport dynamo model are that the meridional circulation plays a crucial role in this model and the poloidal magnetic field is generated by the BabcockLeighton (BL) process involving the decay of tilted bipolar sunspots. Bipolar sunspots are assumed to form due to the buoyant rise of the toroidal magnetic flux through the convection zone (Parker 1955b) and their tilts result from the action of the Coriolis force on the rising flux tubes (Choudhuri 1989; D'Silva \& Choudhuri 1993; Fan et al. 1993) leading to Joy's law (Hale et al. 1919). When a tilted pair of bipolar sunspots decays, turbulent diffusion spreads the magnetic flux to produce a poloidal magnetic component (Babcock 1961; Leighton 1964). An over-all poloidal field develops from the contributions due to many bipolar sunspots and is advected to the poles by the meridional circulation, which is poleward in the upper layers of the convection zone. The polar magnetic field of the Sun is built up in this process.

The BL process - which involves the production of tilted bipolar sunspot pairs and the generation of the poloidal field from their decay - is an inherently 3D process and can be modeled in 2D only through drastically

ghazra@physics.iisc.ernet.in,

arnab@physics.iisc.ernet.in \& miesch@ucar.edu simplified crude approximations (Choudhuri \& Hazra 2016). Still an understanding of how the poloidal field builds up by the BL process historically came from two distinct classes of $2 \mathrm{D}$ theoretical models: the $2 \mathrm{D}$ flux transport dynamo model and the surface flux transport (SFT) model. In the 2D flux transport dynamo model, we average over the azimuthal direction $\phi$ and solve the axisymmetric dynamo equation in the $r-\theta$ plane. On the other hand, in the SFT model, we focus our attention only on the $B_{r}$ component of the magnetic field at the solar surface spanned by the $\theta-\phi$ coordinates and study its evolution on this surface under the joint action of diffusion, meridional circulation and differential rotation. Neither of these approaches provides a fully satisfactory depiction of the BL process and each approach has its own limitations.

If a tilted bipolar sunspot pair at the solar surface is averaged over the azimuthal direction $\phi$, then we get two rings of opposite magnetic polarity at slightly different latitudes. Durney $(1995,1997)$ advocated the development of the flux transport dynamo model by using such double rings as the source of the poloidal component. However, a more popular approach has been to introduce an $\alpha$-coefficient reminiscent of the $\alpha$-effect of the mean field dynamo theory (Parker (1955a); Steenbeck et al. (1966); Choudhuri (1998), Chapter 16), although this now has a completely different interpretation. The source term of the poloidal field is taken as the product of this $\alpha$-coefficient and the toroidal field that has risen from the tachocline due to magnetic buoyancy. Choudhuri \& Hazra (2016) review how different authors achieve this, with references to the original papers. Nandy \& Choudhuri (2001) showed that the double ring approach and the treatment through $\alpha$-coefficient give qualitatively similar results, although Muñoz-Jaramillo et al. (2010) argued that the double ring approach is more realistic. 
In any case, the 2D kinematic dynamo models do not give a detailed picture of how the poloidal field builds up from the contributions of many individual bipolar sunspot pairs, since such pairs get smeared over when we average over the azimuthal direction. Also, as most of these dynamo models rely on a mean field approach, flux tubes or sunspots are not handled properly in these models (Choudhuri 2003).

Starting from the pioneering work of Wang et al. (1989b,a), the surface flux transport (SFT) model has been made more sophisticated in several recent studies (van Ballegooijen et al. 1998; Schrijver et al. 2002; Baumann et al. 2004, 2006; Cameron et al. 2010; Jiang et al. 2014a; Upton \& Hathaway 2014; Jiang et al. 2015). In this model, recently reviewed by Jiang et al. (2014b), one can study in detail how individual sunspot pairs contribute in building up the poloidal field and can address such questions as to how this process depends on such factors as the latitudinal positions of the sunspot pairs and the distribution in their tilt angles. The main limitation of this model is that several important aspects of physics get left out by ignoring the vectorial nature of the magnetic field and by not including any subsurface processes. By studying the time evolution of an axisymmetric poloidal field, Dikpati \& Choudhuri (1994, 1995 ) and Choudhuri \& Dikpati (1999) showed that the subduction of the poloidal field by the meridional circulation sinking underneath the surface at the polar region plays an important role in the dynamics of the magnetic field. Since this process cannot be included in the SFT models, flux of $B_{r}$ tends to get piled up in the polar regions and has to be neutralized by flux of the opposite sign advected there. If additional flux of the opposite sign is not brought there, then the polar field may reach an asymptotic value as seen in Figure 6 of Jiang et al. (2014a). When one tries to model several successive cycles through an SFT model, one may get a 'secular drift' of the polar field if the flux of the succeeding cycle is unable to properly neutralize the polar flux of the preceding cycle, as seen in Figure 1 of Baumann et al. (2006). A way of fixing this problem proposed by Baumann et al. (2006) involves adding an ad hoc decay term corresponding to the radial diffusion not included in the SFT model. In spite of the tremendously important historical role the SFT model has played in elucidating the BL process, this model has the inherent limitation that it cannot adequately handle the magnetic field dynamics in the Sun's polar region.

We believe that the next step forward is the 3D kinematic flux transport dynamo model. In this model, the fluid motions (differential rotation, meridional circulation) are specified and the evolution of the magnetic field is calculated in 3D. Such a model has the promise of incorporating the attractive features of both the $2 \mathrm{D}$ flux transport dynamo model and the SFT model, while being free from the limitations of both these models. It can handle the $\mathrm{BL}$ process much more realistically than the 2D flux transport dynamo model, where we average over the azimuthal direction and cannot include tilted bipolar sunspots properly. On the other hand, this model incorporates the vectorial nature of the magnetic field and the subsurface processes which are left out in the SFT models.

Efforts of constructing 3D kinematic flux transport dy- namo models began only within the last few years. In a landmark paper, Yeates \& Muñoz-Jaramillo (2013) developed a method of treating the buoyant rise of a flux tube in their 3D dynamo model by simultaneously applying a radially outward and a vortical velocity to a localized part of an azimuthal flux tube at the bottom of the convection zone. Although they did not present a selfexcited dynamo solution, they simulated a solar cycle by incorporating bipolar sunspot eruptions by this method at the actual locations where bipolar sunspots were observationally seen. Miesch \& Dikpati (2014) succeeded in producing a self-excited dynamo by identifying the locations (in latitude and longitude) at the bottom of the convection zone where the toroidal field was the strongest (in the theoretical model) and then putting tilted bipolar sunspot pairs above those locations with loop-like magnetic structures both above and below the surface (by using an algorithm which they named SpotMaker). More details of this model have been given by Miesch \& Teweldebirhan (2016). After a part of the toroidal flux tube rises to the surface to produce bipolar sunspots, the magnetic field underneath these sunspots has to be detached at some stage from the bottom of the convection zone before the magnetic flux of the sunspots is dispersed freely by diffusion and carried poleward by the meridional circulation (Longcope \& Choudhuri 2002). Our lack of understanding of this process is the main difficulty in constructing realistic 3D dynamo models at the present time. Presumably, the approach of Yeates \& Muñoz-Jaramillo (2013) captures the physics of the early phase soon after the bipolar sunspots emerge, whereas the approach of Miesch \& Dikpati (2014) is more appropriate for the later phase when the magnetic field below the sunspot pairs has become detached from the bottom of the convection zone.

The aim of the present paper is to use a modified version of the model of Miesch \& Dikpati (2014) to study the build-up of the Sun's polar magnetic field by the BL process in more detail. The model of Miesch \& Dikpati (2014) uses values of parameters (such as turbulent diffusion) which are probably not very realistic for the Sun. We use the same dynamo code named as STABLE (i.e. Surface flux Transport And Babcock LEighton Model) to first construct a model of the solar dynamo based on more realistic values of parameters and then use this model for our study. Since the BL process has been studied most extensively by the SFT models, we especially address the question whether the insights gained about various aspects of this process from the SFT models are borne out by the 3D model or have to be revised significantly. We shall see that the accumulation of magnetic flux at the poles seen in the SFT models does not occur when the low-latitude advection and emergence of oppositely-signed radial flux is taken into account. Thus, the problem of 'secular drift' is automatically eliminated. One insight from the SFT models is that the fluxes of leading sunspots at lower latitudes get canceled across the equator and the fluxes from the following sunspots are then advected to the poles, building up a dipole moment of the Sun. We shall see that this insight also will have to be modified significantly. SFT models indicate that even a few large sunspot pairs with anti-Hale or wrong polarity (i.e. opposite of what is expected of sunspot pairs in that cycle) may have significant effect 
on the polar field (Jiang et al. 2015). We shall be able to study this effect more realistically in our 3D model.

After discussing the mathematical formulation of the problem in the next section, the standard model of the solar dynamo which we shall use is presented in $\S 3$. Then the build-up of the polar field is studied in $\S 4$, while the effects of large anti-Hale sunspot pairs are discussed in $\S 5$. Our conclusions are summarized in $\S 6$.

\section{MATHEMATICAL FORMULATION}

In this section, we explain the basic formulation of the STABLE dynamo model which is first reported in Miesch \& Dikpati (2014) and in more detail in Miesch \& Teweldebirhan (2016). This model is a 3D generalization of the pre-existing axisymmetric 2D flux transport dynamo models and it solves the induction equation in full 3D rotating spherical shell with radius ranges from $r=0.69 R$ to $r=R$ of the Sun:

$$
\frac{\partial \mathbf{B}}{\partial t}=\nabla \times\left(\mathbf{v} \times \mathbf{B}-\eta_{t} \nabla \times \mathbf{B}\right)+S(\theta, \phi, t)
$$

where $\mathbf{v}$ is the velocity field, $\eta_{t}(r)$ is the turbulent diffusion in the solar convection zone, and $S(\theta, \phi, t)$ is the source function which captures the effect of the BL mechanism. As we shall discuss in detail later, the source function $S(\theta, \phi, t)$ is of the nature of an impulsive forcing term which becomes non-zero only at the instants when we allow a bipolar sunspot pair to be put at the solar surface. Though this model is fully $3 \mathrm{D}$ and no axisymmetric assumption is considered, still this model is kinematic and we provide the velocity field motivated from helioseismology and observations. We solve equation (1) using Anelastic Spherical Harmonic (ASH) code (Miesch et al. 2000; Brun et al. 2004). ASH is a well-established pseudospectral code which has been used extensively for 3D solar and stellar convection simulations, instabilities, tachocline confinement and many other aspects of solar and stellar internal dynamics. The ASH code has capability to solve the velocity equation and magnetic induction equation together but for our kinematic model we bypass the velocity equation solver and only solve the induction equation by providing observationally motivated velocity fields. The version of the code used for 3D kinematic dynamo modeling is named STABLE (i.e. Surface flux Transport And Babcock LEighton Model).

The mean velocity field $\mathbf{v}$ in the Sun can be written as the summation of the part from differential rotation $\Omega$ and the meridional circulation $v_{p}$. Whereas the exact differential rotation is mapped from helioseismology quite well (Schou et al. 1998), the structure of the meridional circulation in the solar convection zone is still under study. Recently, different helioseismology groups have reported substantially different structures of the meridional circulation in the solar convection zone (Schad et al. 2013; Zhao et al. 2013; Rajaguru \& Antia 2015). In response to these observational claims, Hazra et al. (2014) carried on calculations with different types of meridional circulation structure and showed that flux transport dynamo works quite well as long as there is an equatorward flow at the bottom of the convection zone. Recently, Karak \& Cameron (2016) showed that in the presence of appropriate profile of downward pumping in the solar convection zone, the flux transport solar dynamo works
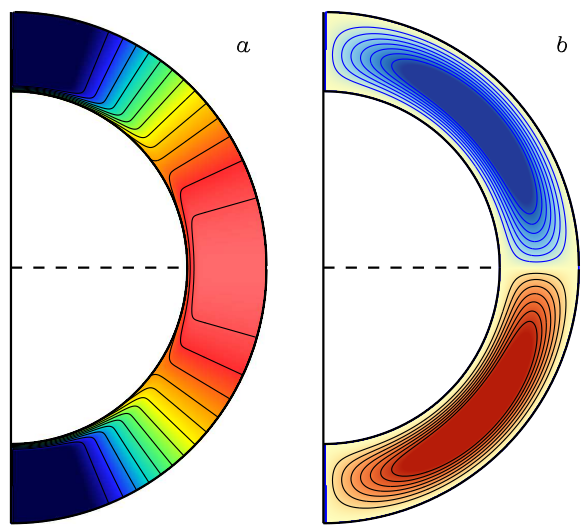

FIG. 1.- (a). Differential rotation profile with color table ranging from $350-480 \mathrm{nHz}$ from blue to red; (b) streamlines for the meridional flow. Blue and red contours show the poleward flow at the surface and an equatorward flow at the bottom of the convection zone in northern and southern hemisphere respectively. The amplitude of the meridional circulation is taken as $20.40 \mathrm{~m} / \mathrm{s}$ on the surface and $1.64 \mathrm{~m} / \mathrm{s}$ at the lower convection zone.
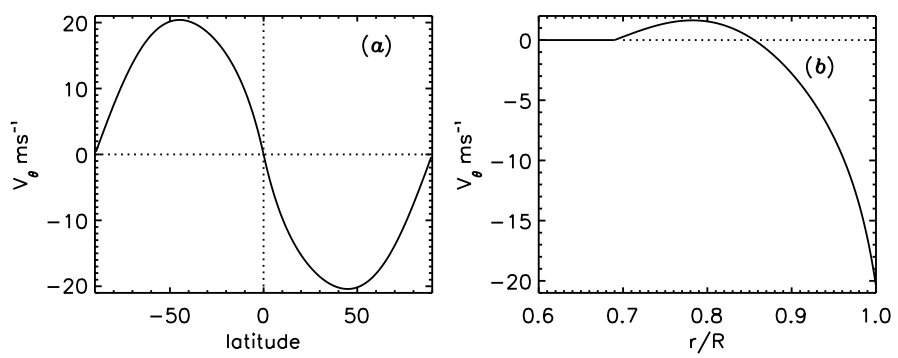

FIG. 2.- Variation of latitudinal component of meridional circulation $V_{\theta}$ with latitude on the surface (a) and with radius at $45^{\circ}$ latitude (b).

with even shallow meridional circulation. Since there is still no compelling reason (Rajaguru \& Antia 2015) to give up the simple single-cell profile of meridional circulation used by many previous authors (Chatterjee et al. (2004), Miesch \& Dikpati (2014)), we use a single cell profile of the meridional circulation having a poleward flow at the surface and an equatorward return flow at the bottom of the convection zone. The stream function corresponding to the meridional circulation which we use here is

$$
\begin{aligned}
\psi r \sin \theta=\psi_{0}(r- & \left.R_{p}\right) \sin \left[\frac{\pi\left(r-R_{p}\right)}{\left(R-R_{p}\right)}\right]\left\{1-e^{-\beta_{1} r \theta^{\epsilon}}\right\} \\
& \times\left\{1-e^{\beta_{2} r(\theta-\pi / 2)}\right\} e^{-\left(\left(r-r_{0}\right) / \Gamma\right)^{2}}
\end{aligned}
$$

with $\beta_{1}=0.3 \times 10^{-10} \mathrm{~cm}^{-1}, \beta_{2}=0.5 \times 10^{-10} \mathrm{~cm}^{-1}$, $\epsilon=2.0000001, r_{0}=\left(R-R_{b}\right) / 4.0, \Gamma=3.5 \times 10^{10} \mathrm{~cm}$, $R_{p}=0.69 R$. The value of $\psi_{0}$ determines the amplitude of the meridional circulation. On taking $\psi_{0}=12.0$, the poleward flow near the surface at mid-latitudes peaks around $v_{0}=20.40 \mathrm{~m} \mathrm{~s}^{-1}$. The contour plot for the meridional circulation is shown in figure $1(\mathrm{~b})$ and the variation of $V_{\theta}$ with latitude on the surface and variation $V_{\theta}$ with radius at mid-latitude $\left(45^{\circ}\right)$ are shown in figure 2(a) and 2(b) respectively. For differential rotation we have used the analytical formula given in (Dikpati \& Charbonneau 1999) which is a good fit to the observa- 


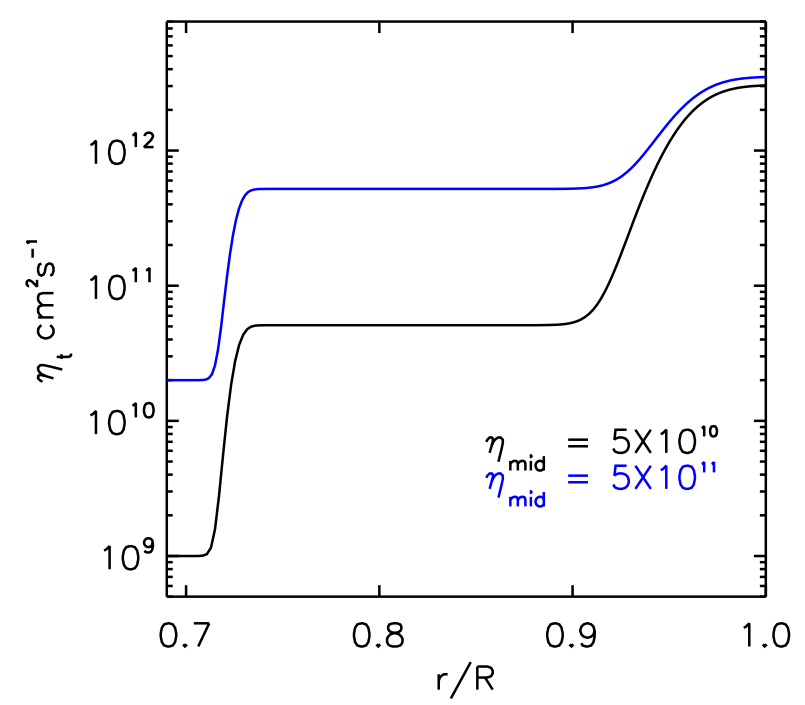

FIG. 3.- High diffusivity profile used for most of our simulation is shown in blue solid line and the profile used for advectiondominated regime is shown in black solid line.

tional data (Figure 1(a)).

Turbulent diffusivity is another important parameter. After the BL process generates the poloidal field near the solar surface, it has to reach the tachocline where the differential rotation acts on it to produce the toroidal field. This can happen in two ways. The poloidal field may first be advected by the meridional circulation to the pole and then underneath the surface to the midlatitude tachocline from where the first sunspots of cycle rise. The time scale for this is close to 20 years for a reasonable profile of the meridional circulation. The second possibility is that the poloidal field diffuses from the surface to the bottom of the convection zone to be acted upon by the differential rotation of the tachocline. The Green's function for the diffusion equation suggests that the diffusion time across a length $L$ is $L^{2} / 4 \eta_{t}$ (see, for example, Parker (1979), p. 32). If the turbulent diffusivity within the convection zone is assumed to be $5 \times 10^{11}$ $\mathrm{cm}^{2} \mathrm{~s}^{-1}$ as we shall do, then this diffusion time turns out to be about 7 years if we take $L$ to be the thickness of the convection zone. The value of the turbulent diffusivity determines whether the poloidal field is transported across the convection zone primarily by meridional circulation or by turbulent diffusion, and the behavior of the dynamo is very different in the two situations (Jiang et al. 2007; Yeates et al. 2008). Over the years, we have got more and more evidence that the turbulent diffusivity has to be sufficiently high to make the poloidal field transport primarily by diffusion in order to explain many aspects of the solar cycle, such as the dipolar parity (Chatterjee et al. 2004; Hotta \& Yokoyama 2010), the lack of significant hemispheric asymmetry (Chatterjee \& Choudhuri 2006; Goel \& Choudhuri 2009), the observed correlation between the polar field at the cycle minimum and the strength of the next cycle (Jiang et al. 2007), the Waldmeier effect (Karak \& Choudhuri 2011). Such a value of turbulent diffusivity is also consistent with mixing length arguments (Parker (1979), p. 629) and the theory of mean flows (Miesch et al. 2012).

In most of the SFT models, a constant diffusivity (2.5-
$3.0) \times 10^{12} \mathrm{~cm}^{2} \mathrm{~s}^{-1}$ on the surface is used (Jiang et al. 2014b). The turbulent diffusivity is expected to be less within the convection zone and falls drastically at its bottom where convection is less vigorous. Except when stated explicitly otherwise, the calculations of this paper assume the diffusivity to be given by

$$
\begin{aligned}
\eta_{t}=\eta_{c}+ & \frac{\eta_{\text {mid }}}{2}\left[1+\operatorname{erf}\left(2 \frac{r-r_{d a}}{d_{a}}\right)\right] \\
+ & \frac{\eta_{t o p}}{2}\left[1+\operatorname{erf}\left(2 \frac{r-r_{d b}}{d_{b}}\right)\right]
\end{aligned}
$$

where $\eta_{c}=2 \times 10^{10} \mathrm{~cm}^{2} \mathrm{~s}^{-1}, \eta_{\text {mid }}=5 \times 10^{11}$ $\mathrm{cm}^{2} s^{-1}, r_{d a}=0.725 R, r_{d b}=0.956 R$, and $d_{b}=0.05 R$. In Figure 3 we have shown the diffusivity profile by the blue solid line. For comparison, the diffusivity profile used by Miesch \& Dikpati (2014) is shown by the black solid line. It may be noted that some groups (Dikpati \& Charbonneau 1999; Dikpati \& Gilman 2006), over the years, used a rather low value of diffusivity. As seen in Figure 3, Miesch \& Dikpati (2014) and Miesch \& Teweldebirhan (2016) followed these authors in using a diffusivity which was, within the body of the convection zone, about one order of magnitude smaller than what we are using. In our case the diffusivity $\eta_{\text {mid }}$ in the convection zone is $5 \times 10^{11} \mathrm{~cm}^{2} \mathrm{~s}^{-1}$, whereas Miesch \& Dikpati (2014) and Miesch \& Teweldebirhan (2016) use $5 \times 10^{10} \mathrm{~cm}^{2} \mathrm{~s}^{-1}$. Such a lower value of diffusivity would make the diffusion time across the convection zone of the order of 70 years and the advection by the meridional circulation would clearly be the dominant process for the transport of the poloidal field. Miesch \& Dikpati (2014) presented a self-excited dynamo solution for this situation. For the value of diffusivity we are using, the diffusion across the convection zone is the primary process for bringing the poloidal field from the solar surface to the tachocline. We believe that we are the first to obtain a self-excited 3D kinematic dynamo solution for this case, which we contend is closer to reality.

We now discuss how the source term $S(\theta, \phi, t)$ in (1) is specified with the help of the SpotMaker algorithm to treat the BL process. This algorithm is mainly a 3D generalization of the Durney's double ring algorithm (Durney 1995, 1997). In this algorithm, two suitable opposite-polarity spots are placed on the surface of the Sun in response to the dynamo-generated field near the base of the convection zone and then they are allowed to decay in the presence of mean flows (meridional circulation and differential rotation) and diffusivity. The first aim of this algorithm is to find out the suitable position for these spots to be placed on the surface. To do so, we calculate the mean toroidal flux $\bar{B}(\theta, \phi)$ near the bottom of the convection zone averaged over the tachocline thickness(Miesch \& Teweldebirhan 2016) and find out where this field is crossing the threshold value $B_{t}$. It is believed that if magnetic fields near the bottom of the convection zone are stronger than the threshold value $B_{t}$ then they become magnetically buoyant and create the bipolar sunspots on the surface (Parker 1975). So the latitude and longitude of the spot pair is chosen randomly from all grid points where the mean toroidal flux exceeds $B_{t}$, subject to a mask that suppresses spots at high latitudes. When we are able to find the $\theta_{s}$ and $\phi_{s}$ where the 
dynamo-generated toroidal field is more than the threshold value $B_{t}$, we put two spots on the surface at that position. Once the position of the bipolar sunspots is decided, the next step is to specify the magnetic field there, by putting some tilt angle between the two sunspots according to Joy's law. For that we use the polynomial profile as given in Miesch \& Dikpati (2014) and for tilt angle we follow the procedure given in Stenflo \& Kosovichev (2012). We choose the tilt angle to be $\delta=32.1^{\circ} \cos \theta$. We do not want to put these spots at each time step of our simulation. There are always certain time differences between the appearances of different sunspot groups. So we have used a time delay probability density function which allows us to put successive sunspot pairs having a random time delay between their appearances (Miesch \& Teweldebirhan 2016). Another thing we should mention here is that, as seen in the observed butterfly diagram, sunspots are found mostly on the lower latitudes and in our model we artificially suppress the sunspot formation at higher latitudes using some masking function as given in equation (3) of the Miesch \& Dikpati (2014). The flux content in the spots and the strength of the radial field are chosen based on the dynamo-generated field $\bar{B}$ and the observed strength of the sunspots as given below.

$$
\Phi=2 \alpha_{\text {spot }} \frac{\left|\hat{B}\left(\theta_{s}, \phi_{s}, t\right)\right|}{B_{q}} \frac{10^{23}}{1+\left(\hat{B}(\theta, \phi) / B_{q}\right)^{2}} \mathrm{Mx}
$$

where $\hat{B}=g(\theta) \bar{B}$ is the toroidal field after using the masking function $g(\theta)$ to suppress sunspots at high latitude and $B_{q}$ is the quenching field strength. Here, $\alpha_{\text {spot }}$ is the parameter which determines whether the dynamo will be sub-critical or super-critical. Our ultimate aim would be to make the dynamo work with $\alpha_{\text {spot }}=1$ so that the flux in a particular BMR will have a value of $10^{23}$ $\mathrm{Mx}$ as observed in case of the subsurface field strength equivalent to the quenching field strength. But if the subsurface field at the bottom of the convection zone is not close to the quenching field, then we have to increase the value of $\alpha_{\text {spot }}$ in order to get a working dynamo with bigger spots. In case of the diffusion-dominated dynamo, we are able to get a working dynamo with $\alpha_{\text {spot }}=100$. While creating sunspot pairs by the SpotMaker algorithm, once the total flux is fixed by (4), we have the freedom of selecting either the magnetic field strength or the size. We choose the magnetic field strength inside the sunspots to be $3000 \mathrm{G}$, which fixes the size.

Since we are solving magnetic fields in a $3 \mathrm{D}$ spherical shell, so we must have to specify the subsurface structure of the sunspots which are put on the surface using the SpotMaker algorithm. As it is argued by Longcope \& Choudhuri (2002) and Schüssler \& Rempel (2005) that the sunspots get quickly disconnected from the parent flux tube, we make a very simple potential field approximation for the sunspot fields (see Figure 2(a,b) of Miesch \& Teweldebirhan (2016)). We ensure that the radial field becomes zero at some penetration depth $(r=0.90 R)$ and it is equal to the imposed sunspot field at the surface $(r=R)$. For the upper boundary condition, we take the magnetic field to be radial at the solar surface. Throughout our simulation we use $N_{r}=200, N_{\theta}=256$ and $N_{\phi}=$ 512. All of the cases where we show the field lines above the solar surface $(r=R)$ are the extrapolated fields using a free potential approximation.

\section{OUR REFERENCE MODEL}

Now we present a self-excited solution from our reference model with parameters as prescribed in the previous section. To the best of our knowledge, this is the first self-excited 3D kinematic dynamo solution in which the diffusivity has been assumed sufficiently high to make sure that the poloidal field is transported from the surface to the tachocline primarily by diffusion. The earlier results presented by Miesch \& Dikpati (2014) and Miesch \& Teweldebirhan (2016) were obtained with a diffusivity one order of magnitude smaller and the transport of the poloidal field was due to the meridional circulation.

Figure 4 shows a butterfly diagram obtained by putting the longitude-averaged $B_{r}$ in a time-latitude plot. One clearly sees the butterfly diagram of sunspots at lower latitudes and the poleward advection of the magnetic field by the meridional circulation at higher latitudes. Superficially, this resembles Figure 6(a) of Miesch \& Teweldebirhan (2016), although our solution is for the diffusion-dominated case in contrast to the solution of Miesch \& Teweldebirhan (2016) obtained for the case dominated by advection due to the meridional circulation. The differences between the two cases become clear on looking at the distribution of the magnetic field. Figure 5 shows the evolution of the toroidal and the poloidal fields during a cycle. Comparing with Figure 8 of Miesch \& Teweldebirhan (2016), we see some obvious differences. In the solution of Miesch \& Teweldebirhan (2016), the oppositely directed toroidal fields on the two sides of the equator almost pressed against each other. Due to the low diffusivity, there would not be much diffusion of the toroidal field even when two opposite bands are brought so close to each other. In our model with higher diffusivity, however, there would be more diffusion of the toroidal field across the equator, making sure that the bands of concentrated opposite polarity are kept somewhat apart, as seen in Fig. 5.

The solar magnetic field is predominantly dipolar. One requirement of a theoretical solar dynamo model is that it should have dipolar parity. We have run our reference model for several cycles to ensure that the dipolar parity persisted. One important question is, under what circumstances we would expect dipolar parity. This question has been studied thoroughly by Chatterjee et al. (2004) and Hotta \& Yokoyama (2010) for the 2D kinematic dynamo model. A full study of this question requires running the code for many different combinations of parameters and running it for a large number of cycles for each such combination. This would require a huge amount of computer time for the 3D model. Because of the limited computer time available to us, we have not been able to study this question exhaustively. However, we have made a limited number of runs to explore the issue of parity a little bit. The best way to look at the issue of parity is to make a butterfly diagram of longitudinally averaged $B_{\phi}$ at the bottom of the convection zone, as done in Figure 7(a) of Chatterjee et al. (2004). In Figure 6(a) we show such a plot for our reference model, whereas Figure 6(b) shows a similar plot for the case in which the value of diffusivity within the convection zone has been changed from $5 \times 10^{11} \mathrm{~cm}^{2} \mathrm{~s}^{-1}$ to $7 \times 10^{11} \mathrm{~cm}^{2}$ $\mathrm{s}^{-1}$ while keeping all the other parameters exactly the 


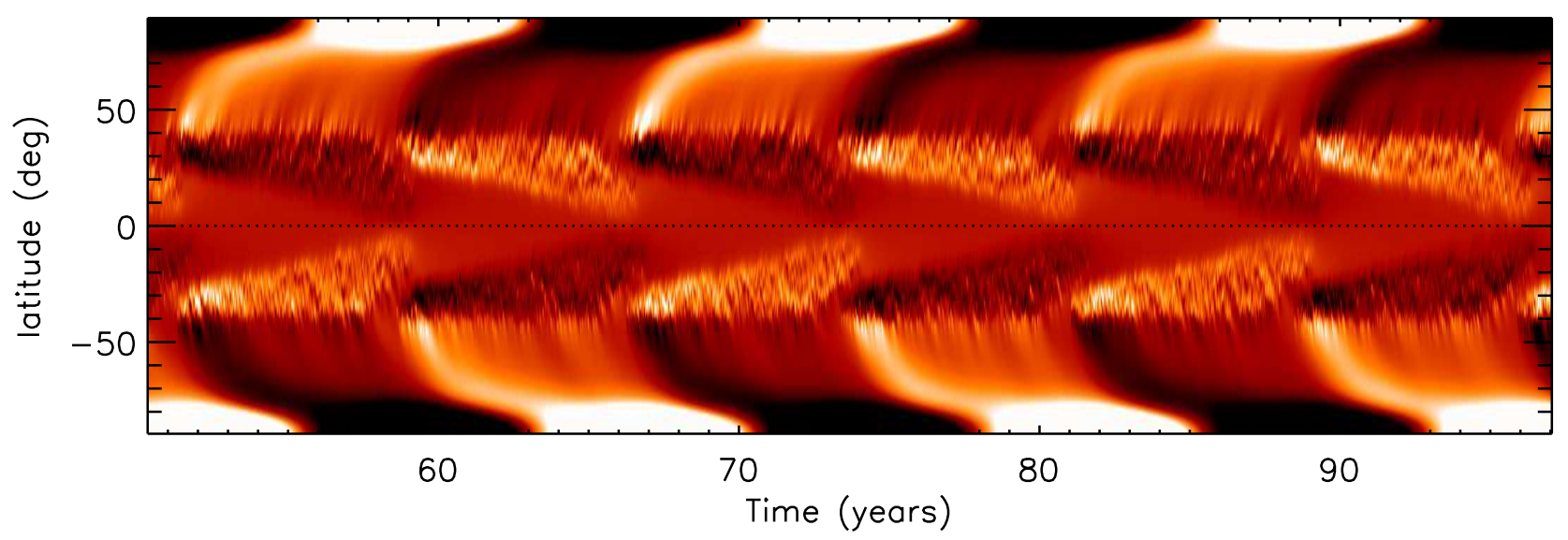

FIG. 4. - Time-latitude plot of longitudinally averaged radial magnetic field on the surface $(r=R)$ of the Sun. Color table is set at \pm 10 $\mathrm{kG}$.
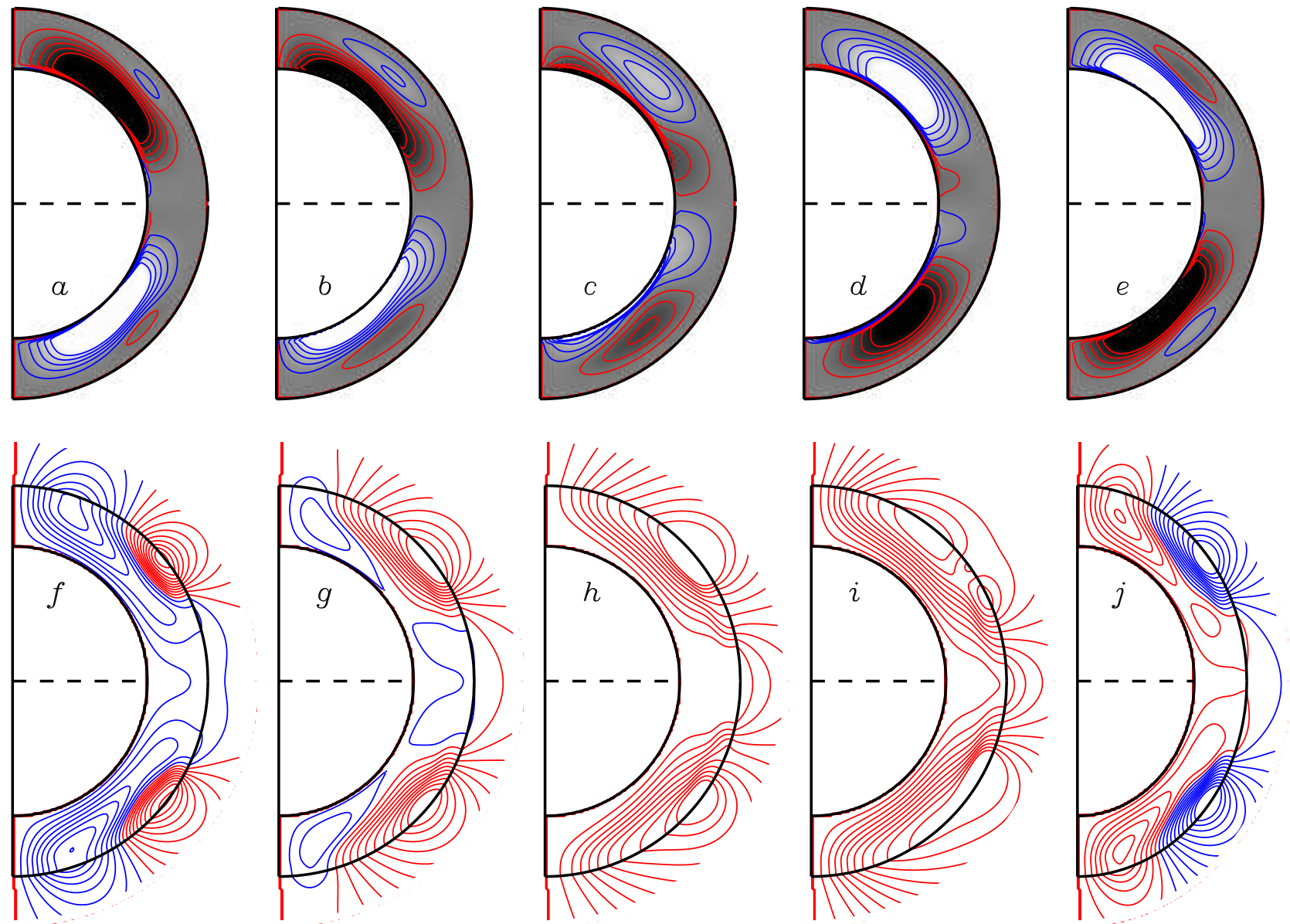

FIG. 5.- Mean toroidal and poloidal field lines are shown for a particular solar cycle at five different time: $t=60.0$ (a), (f), 62.0 (b), $(\mathrm{g}), 64.0(\mathrm{c}),(\mathrm{h}), 66.0(\mathrm{~d}),(\mathrm{i})$ and 68.0 years $(\mathrm{e}),(\mathrm{j})$. Frames (a)-(e) show mean toroidal fields with red and blue lines indicating eastward and westward field respectively. Filled color also represents the mean toroidal fields. Color table is set in this case at $\pm 500 \mathrm{kG}$. Frames (f)-(g) represent poloidal magnetic potential with potential field extrapolation above the surface (upto $r=1.25 R$ ) where red and blue lines represent clockwise and anticlockwise directions. The maximum and minimum contour level is set corresponding to the poloidal field strength of $\pm 49 \mathrm{kG}$ 
same as in our reference model. We clearly see in Figure 6 (b) that the nature of the solution is changing from a dipolar parity to a quadrupolar parity.

The surprising fact is that we now seem to get a result which is the opposite of what Chatterjee et al. (2004) and Hotta \& Yokoyama (2010) obtained for the 2D kinematic dynamo model. These authors found that the dipolar parity is preferred on increasing the diffusivity, whereas we now are finding the opposite of that. Let us look at the physics of the problem. In a dipolar mode, the poloidal magnetic field lines connect across the equator, whereas the toroidal field on the two sides of the equator has to be directed oppositely. In order for this to happen, we need diffusivity to have a big effect on the poloidal field, but not much effect on the toroidal field. In the model of Chatterjee et al. (2004), any strong toroidal field within the convection zone was removed by magnetic buoyancy and the toroidal field at the bottom of the convection zone was also depleted continuously to account for flux loss due to magnetic buoyancy. As a result, the toroidal magnetic field near the equator was naturally weak and the effect of diffusion was more important on the poloidal field than on the toroidal field. This ensured that higher diffusivity favored the dipolar mode. In the present calculation, the situation is rather different. The strong parts of the toroidal field are now allowed to hover in the middle of the convection zone and near the equator. On increasing diffusivity, the quadrupolar mode in which the toroidal field on the two sides of the equator has the same sign is favored. Here, we should mention that the magnetic pumping can play a very important role to promote dipolar parity (Guerrero \& de Gouveia Dal Pino 2008). Another point to note is that Chatterjee et al. (2004) used a lower diffusivity of the toroidal field compared to the poloidal field, to account for the quenching of turbulent diffusion due to the stronger toroidal magnetic field. This could be done in a 2D mean field model in which the evolution equations for the toroidal and poloidal fields neatly separate out, and one could use different values of diffusivity in the two equations. Since it is not possible to do this in a $3 \mathrm{D}$ non-axisymmetric model in which the equations for the toroidal and poloidal components do not split in this way, we have used a single diffusivity. It is possible that the weaker diffusivity of the toroidal field in Chatterjee et al. (2004) helped in producing a dipolar parity by allowing toroidal fields of opposite sign to exist on the two sides of the equator more easily. One way of capturing the physics of this in a $3 \mathrm{D}$ non-axisymmetric model may be to include a quenching of turbulent diffusivity in the regions of strong magnetic field. We plan to explore the effect of this in future.

With these two opposite results at hand, one crucial question is: which of the two results is closer to reality? Although we cannot assert this with confidence at this stage, we believe that the $2 \mathrm{D}$ kinematic dynamo result that the dipolar parity is preferred on increasing diffusivity is the more appropriate result. Although in this paper we are taking account of the 3D nature of magnetic buoyancy and, in that sense, treating magnetic buoyancy more realistically, we still have not taken account of flux depletion from the convection zone and its bottom in an appropriate way. This is probably one important reason why our results are not matching with the results of previous 2D models (Chatterjee et al. 2004; Hotta \& Yokoyama 2010). We are right now exploring possible schemes to take account of the flux depletion due to magnetic buoyancy in a realistic way. We believe that this flux depletion is quite important in the solar dynamo. Choudhuri \& Hazra (2016) found that the Waldmeier effect cannot be reproduced from a theoretical dynamo model unless the flux depletion is taken into account. We have a future plan of incorporating flux loss due to magnetic buoyancy in a realistic way in our 3D kinematic dynamo model and then studying the parity issue more carefully.

Since we are interested in a dynamo solution which has dipolar parity, we have converged on the reference solution presented here. If we decrease diffusivity, then we are led to the case where the meridional circulation provides the main transport mechanism for the poloidal field. On the other hand, if we increase diffusivity, then we obtain the quadrupolar mode. This is what has led us to choose the value $5 \times 10^{11} \mathrm{~cm}^{2} \mathrm{~s}^{-1}$ for diffusivity inside the convection zone.

\section{THE BUILD-UP OF THE POLAR FIELD}

After constructing the self-excited dynamo model, we now study how individual sunspot pairs contribute to the building up of the polar field and address the question whether our understanding gained from this study necessitates the revision of some insights we have from surface flux transport (SFT) models. For this study, we shall put individual sunspot pairs on the solar surface by hand and look at the evolution of the magnetic field. In other words, we shall now not try to construct selfexcited periodic solutions, although we shall keep using the same values of different parameters that we had used for constructing the self-excited periodic solution.

We start our simulation by putting a single pair of bipolar sunspots in the northern hemisphere at different emergence angles $\lambda_{\text {emg }}$ and let it evolve under the axisymmetric mean flows and diffusion to see the development of the polar field. We have chosen magnetic flux of $1 \times 10^{22} \mathrm{Mx}$ in each spot and its radius is taken to be 21.71 Mm (somewhat larger than actual sunspot radii, to make the results of the simulation more clearly visible) throughout our simulations. In the next set of our simulations, we shall put two pairs of sunspots symmetrically in the two hemispheres, which have the same amount of flux and radius as in the case of the single pair, to see the effects of cross-equator diffusion of magnetic flux.

\subsection{Polar field from one sunspot pair}

We use the SpotMaker algorithm to put one sunspot pair at latitude $20^{\circ}$ with tilt angle $40^{\circ}$. Then, we allow our code to evolve the magnetic field from this sunspot pair leading to the build-up of the polar field. Figure 7 shows snapshots of $B_{r}$ on the solar surface at different times during the evolution process. This figure can be compared with Figure 6 of Yeates \& Muñoz-Jaramillo (2013). Although Yeates \& Muñoz-Jaramillo (2013) assumed the sunspot pair to be initially connected to the toroidal flux system at the bottom of the convection zone, eventually this connection would be disrupted and the evolution of the magnetic field on the surface due to the sunspot pair in the northern hemisphere appears to be very similar to the evolution that we get by assuming 

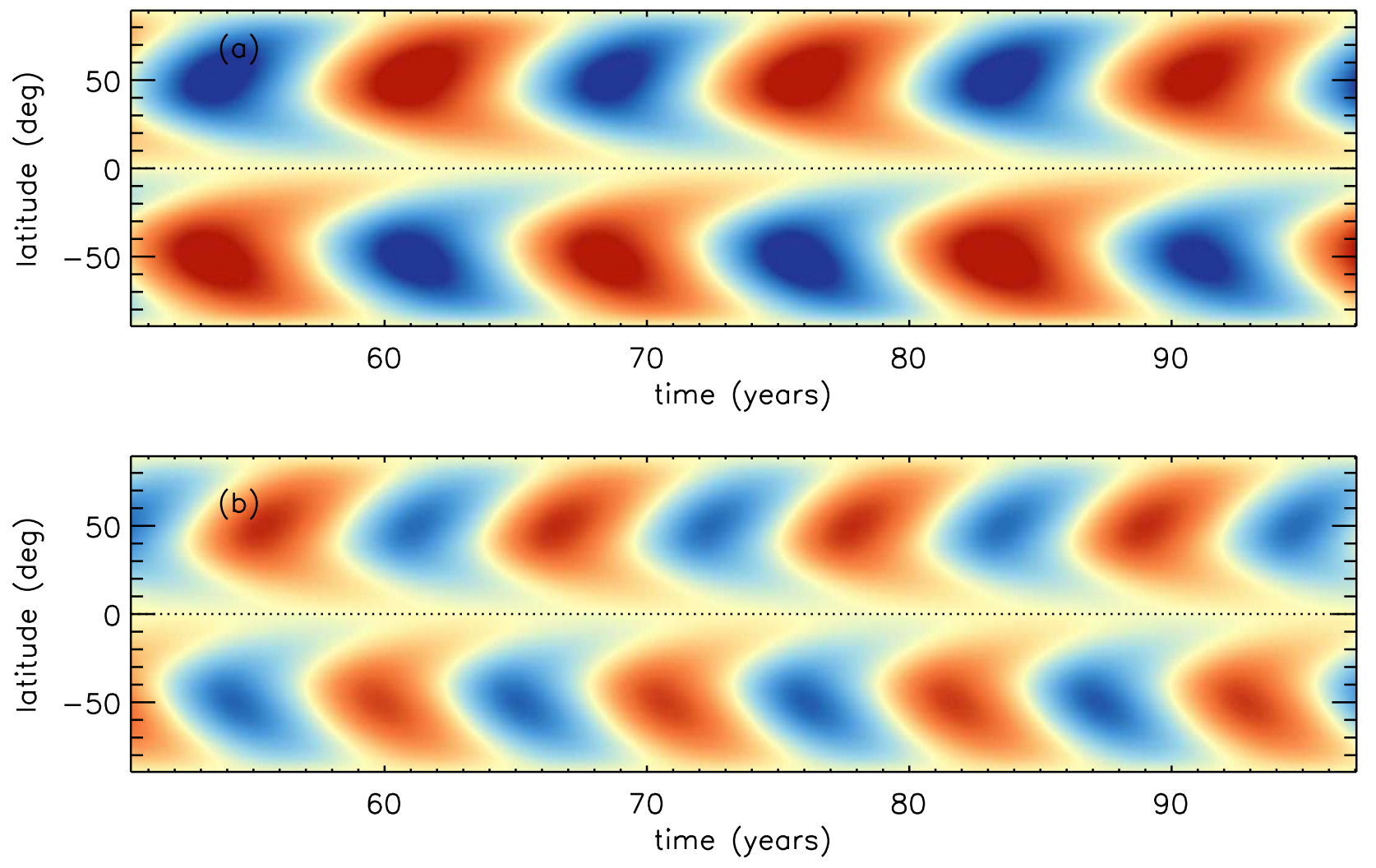

FIG. 6.- Azimuthal averaged toroidal fields $B_{\phi}$ at the bottom of the convection zone $(r=0.71 R)$ for two different values of diffusivity $\eta_{\text {mid }}$ at the convection zone (a) $5 \times 10^{11} \mathrm{~cm}^{2} \mathrm{~s}^{-1}$ and (b) $7 \times 10^{11} \mathrm{~cm}^{2} \mathrm{~s}^{-1}$. Color table is set at $\pm 400 \mathrm{kG}$.

a disconnection from the very beginning. The following sunspot at the higher latitude has the positive polarity and we clearly see that this positive polarity is preferentially transported to the higher latitudes. This positive polarity region gets stretched by the differential rotation into a belt going around the polar axis. When this belt reaches sufficiently high latitude, we see that it is followed by a belt of negative polarity coming from the leading sunspot which was taken at a lower latitude. The meridional circulation takes about 3 years to bring the flux of $B_{r}$ to create a positive patch on the pole surrounded a ring of negative polarity. The formation process of the negative polarity ring is clearly visible in Figure $7(\mathrm{~d})$, but at later times it becomes weaker due to the action of diffusion and is not clearly visible. Since the meridional circulation sinks downward at the polar region, eventually both the positive and negative polarity magnetic fields are advected simultaneously below the surface. This becomes clear from the field line plots shown in Figure 8. At certain instants of time, we have averaged $B_{r}$ and $B_{\theta}$ over the azimuthal direction $\phi$ to obtain the field lines.

It may be noted that the color scale for each plot in Figure 7 is set at \pm maximum values of the magnetic field in each case. This was necessary because the magnetic field becomes weak with time. Had we used the color scale of Figure 7 (a) for all cases shown in Figure 7, then the magnetic field would be completely invisible for the plots at later times. Though the magnetic fields in the sunspot pair remain concentrated for a shorter time than what one may suspect from a casual look at Figure 7, the sunspots in our simulations are nevertheless live longer than real sunspots. This is expected because we have assumed the sizes of sunspots in our calculation to be larger than real sunspots. If sunspots decay by the action of turbulent diffusion, then a simple application of the diffusion equation suggests that the lifetime should go as the square of the size. A hypothetical sunspot 5 times larger than a real sunspot should live 25 times longer than a real sunspot.

It should be kept in mind that $\int \mathbf{B} . d \mathbf{S}$ integrated over the whole solar surface has to be zero at any time (since $\nabla \cdot \mathbf{B}=0)$. This means that, during any time interval, equal amounts of positive and negative magnetic fluxes have to disappear below the surface due to the subduction process. As a result, we see in Figure 7 that the white patch at the pole (representing positive flux) remains there till all fluxes disappear and is not replaced by the poleward migrating dark ring (representing negative flux, which gets subducted along with the positive flux). In the real Sun undergoing successive cycles, the polar field reverses only when fluxes of the following sunspots from the next cycle reach the pole. This subduction process has been seen before in axisymmetric Babcock-Leighton / Flux-Transport dynamo models but has not been well studied within the context of SFT models (though see Cameron et al. (2012); Yeates \& Muñoz-Jaramillo (2013)). It relies on the upward advection of poloidal flux near the equator, which leads to the emergence of oppositely-signed radial field, as shown in Figure $8(f-j)$. This changes the net radial flux through 
the surface in each hemisphere and eats away at the polar field as it is advected poleward. Without this lowlatitude emergence, the subduction of poloidal flux at the poles could not change the net flux through the outer surface.

In the SFT model also, a tilted sunspot pair gives rise to a polar field with the polarity of the following sunspot surrounded by a belt of the opposite polarity. However, since the low-latitude emergence and subsequent subduction of the mean poloidal field is not included in the model, the net flux through each hemisphere can only change by means of cross-equatorial transport and diffusion. In a model of the solar magnetic field dynamics with realistic values of various parameters, usually the diffusion time for neutralizing the opposite magnetic polarities turns out to be much longer than the time for their disappearance due to low-latitude emergence and subduction by the meridional circulation. As we see in Figure 8, the magnetic fields tend to sink below the surface while they diffuse and the disappearance of the magnetic fields at the surface takes place in a time scale shorter than the diffusion time scale. We thus see that the evolution of the polar field in our 3D model is qualitatively different from what it is in the SFT model.

Figure 8 also shows the toroidal field generated in the convection zone. Since the poloidal field has not yet reached the tachocline to be acted upon by the radial differential rotation there, it may be worthwhile to comment how the toroidal field is generated. As soon as we put a sunspot pair on the surface by the SpotMaker algorithm, some toroidal field arises below the surface at once because the magnetic loop connecting the two sunspots below the surface would have a toroidal component. Additionally, more toroidal field is produced by the latitudinal differential rotation within the convection zone. It has been known that the latitudinal differential rotation can play an important role in generating the toroidal field (Guerrero \& de Gouveia Dal Pino 2007). In reality, any strong magnetic field generated within the convection zone is expected to be quickly removed by magnetic buoyancy which is particularly effective within the convection zone (Parker 1975; MorenoInsertis 1983). Since we do not allow magnetic buoyancy to remove the toroidal field in the present version of the code, the toroidal field remains where it is created. However, it may be noted that some fully dynamical simulations suggest persistent rings of toroidal flux within the convection zone (Brown et al. 2010).

Finally, Figure $9(\mathrm{a})$ shows $B_{r}$ (averaged over $\phi$ ) as a function of latitude for different times, whereas Figure 9 (b) shows $B_{r}$ as a function of time at different latitudes. A careful scrutiny of Figure 9(a) makes it clear that the poleward meridional circulation transports the magnetic flux to higher latitudes with time. After about 3 years, the polar field starts building up. It is clear that the polar field becomes much stronger than the fields at mid-latitudes. This is purely a geometrical effect. Since magnetic flux from different longitudes is brought by the meridional circulation to the pole where it converges, it is natural that the magnetic field becomes stronger at the pole. It is also to be noted that we only have the polar field with polarity corresponding to the polarity of the following sunspot at the higher latitude (positive in the present case). Turning to Figure 9(b) now, we first look at the plots corresponding to the mid-latitudes $\left(\approx 30^{\circ}-65^{\circ}\right)$. At a mid-latitude, first the magnetic field corresponding to the polarity of the following sunspot (positive in the present case) is brought by the meridional circulation, followed by the magnetic field with opposite polarity from the leading sunspot (negative in the present case) a little bit later. This is seen in all the mid-latitude plots in Figure 9(b). But we should pay a special attention to the plots for latitudes $15^{\circ}$ and $80^{\circ}$. At the latitude of $15^{\circ}$, the positive magnetic field from the following sunspot is never seen, because the following sunspot appeared at a higher latitude and the meridional circulation transported the flux from its decay toward the pole. On the other hand, at the latitude of $80^{\circ}$, we see only the positive magnetic field which has been brought there from the following sunspot. The negative magnetic field from the leading sunspot forms a negative polarity belt around the pole, as we have already seen, and then it sinks below the surface, so the negative magnetic field is never seen at sufficiently high latitudes. Also, note that, although the peak value of the positive polarity field at $65^{\circ}$ is less than that at $45^{\circ}$ (due to the action of diffusion while the magnetic field is transported to higher latitudes), the positive polarity field again becomes strong at $80^{\circ}$ due to the geometrical effect of converging flow bringing magnetic flux from different longitudes.

\subsection{Polar fields from two sunspot pairs in two hemispheres}

The results of the $3 \mathrm{D}$ model differ more dramatically from the results of the SFT model when we put two pairs of sunspots located symmetrically in the two hemispheres. If the two pairs are sufficiently close to the equator, then magnetic fluxes of the two leading sunspots get canceled by diffusion across the equator. In the SFT model, only the fluxes from the following polarities are advected to the two poles and we eventually get polar patches which are not surrounded by rings of opposite polarity as we found in the case of the single sunspot pair. When the outward spreading of magnetic field from the polar patches by diffusion is eventually balanced by the inward advection by the meridional circulation, we reach an asymptotic steady state in the SFT model, with an asymptotic magnetic dipole which does not change with time. This is seen in Figure 6 of Jiang et al. (2014a). As we shall discuss now, we get a completely different result from the $3 \mathrm{D}$ model.

We see in Figure 10 that polar magnetic patches form with the polarity of the succeeding sunspots. A careful look at this figure, shows some evidence of opposite polarity (i.e. opposite of what we see in the poles) at midlatitudes even when we start from two sunspots placed symmetrically at sufficiently low latitudes in both the hemispheres. The physics of what is happening becomes clear from the plot of field lines shown in Figure 11. After the fluxes from the leading sunspots near the equator cancel, we see that initially we get poloidal field lines spanning both the hemispheres. A look at the field line plots makes it clear that we shall have $B_{r}$ only at high latitudes in the early stages of the evolution of the magnetic field. As the meridional circulation drags the poloidal field toward the poles, we find that eventually the polar fields in the two hemispheres get detached, as a result of which $B_{r}$ again appears at lower latitudes having 

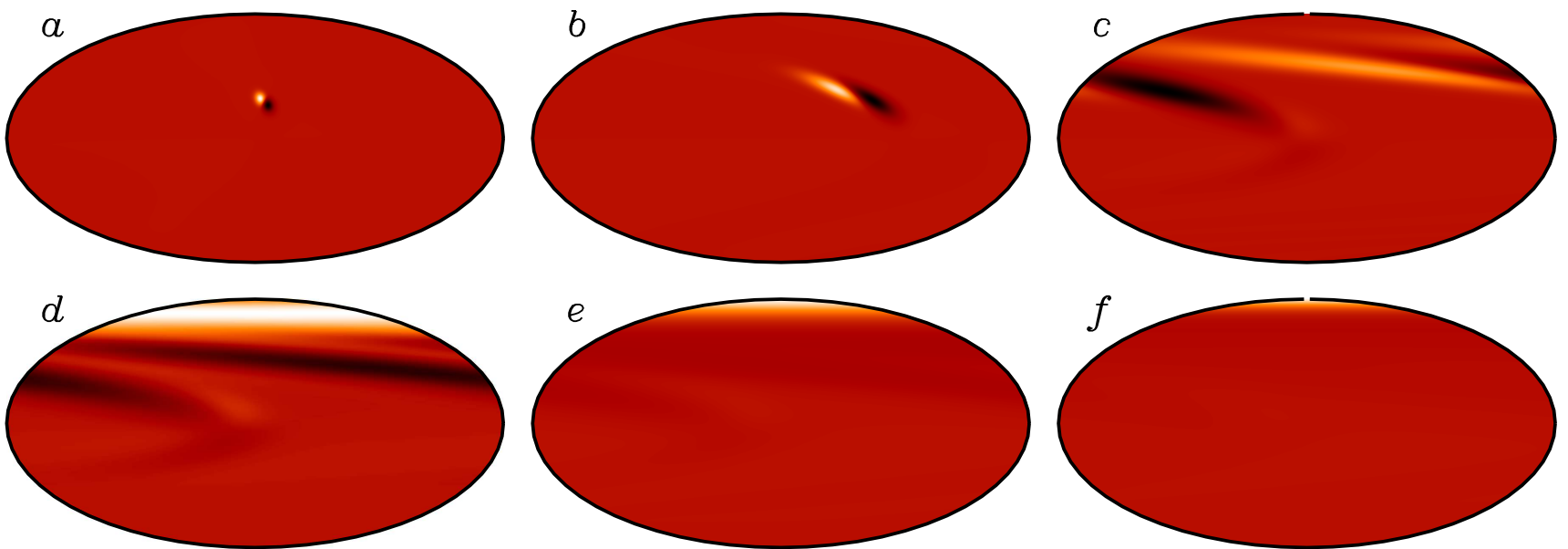

FIG. 7. - Time evolution of radial fields on the surface of the sun with a single pair in the northern hemisphere for (a) $0.025 \mathrm{yr},(\mathrm{b}) 0.25$ yr, (c) $1.02 \mathrm{yr}$, (d) $2.03 \mathrm{yr}$, (e) $3.05 \mathrm{yr}$ and (f) $4.06 \mathrm{yr}$. Here white color shows the outward-going radial field and black color represents inward-going radial field. The color scale is set at \pm maximum values of the magnetic fields for each case. For example $\pm 4.66 \mathrm{G}$ is the color scale for (a) and $\pm 0.10 \mathrm{G}$ is the color scale for (f).
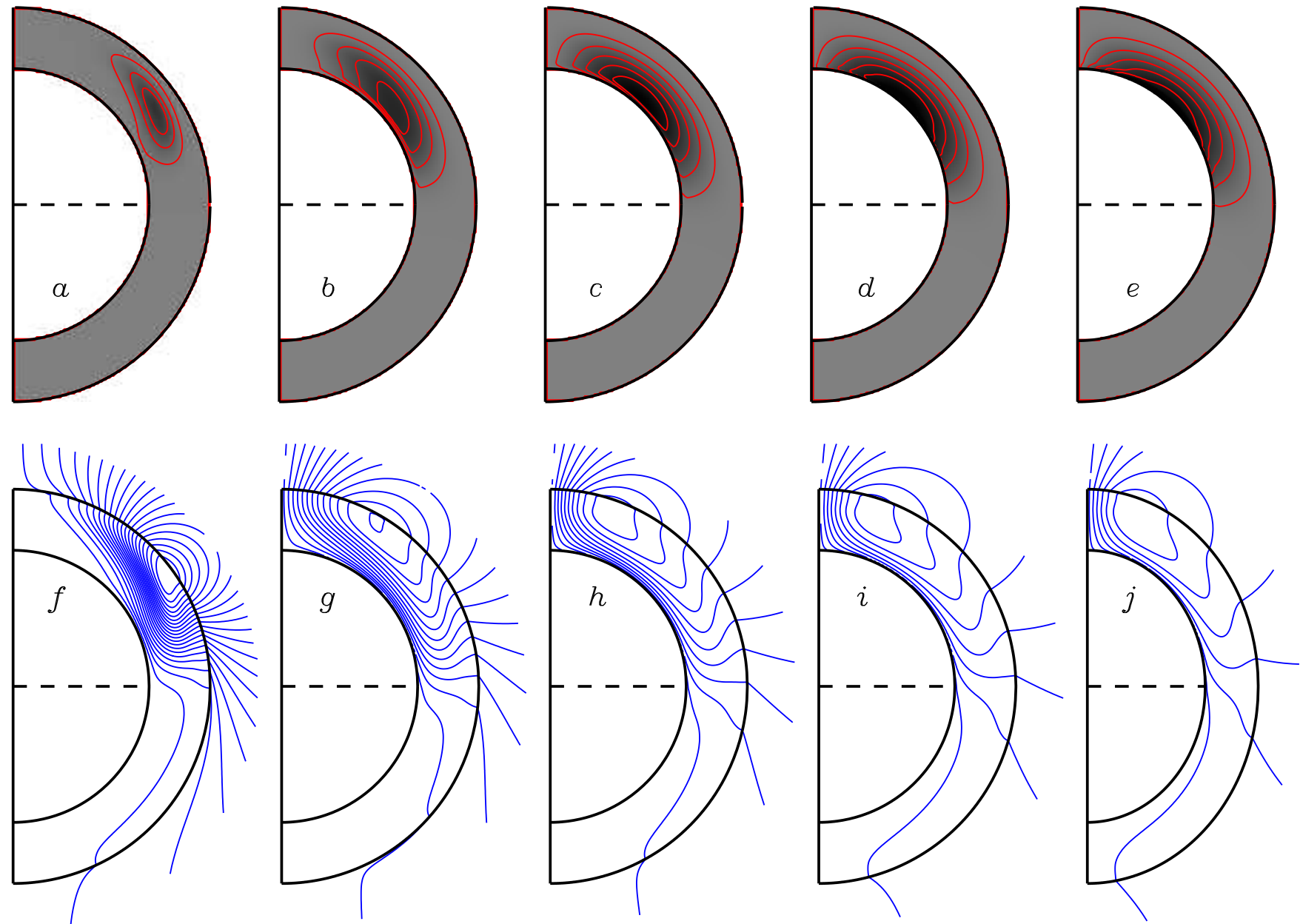

Fig. 8.- Axisymmetric toroidal field lines (a)-(e) and axisymmetric poloidal field lines (f)-(j) are shown for 5 different times. Time spans are $(\mathrm{a}),(\mathrm{f})=1.02 \mathrm{yr},(\mathrm{b}),(\mathrm{g})=3.05 \mathrm{yr},(\mathrm{c}),(\mathrm{h})=5.08 \mathrm{yr},(\mathrm{d}),(\mathrm{i})=7.11 \mathrm{yr}$ and $(\mathrm{e}),(\mathrm{j})=9.15$ yr. Frames $(\mathrm{a})-(\mathrm{e})$ represent $\left\langle B_{\phi}\right\rangle$ (azimuthal averaged) with red and blue indicating eastward and westward fields respectively. Filled contour also represents the mean toroidal fields. Here color scale is set at \pm 1.5 G. Frames (f)-(j) represent the square root of poloidal magnetic potential with potential field extrapolation above the surface (up to $r=1.25 R$ ) and blue color contours denote the clockwise direction of the field. Maximum and minimum contour levels are set corresponding to potential field strength of $\pm 0.3 \mathrm{G}$ respectively. 

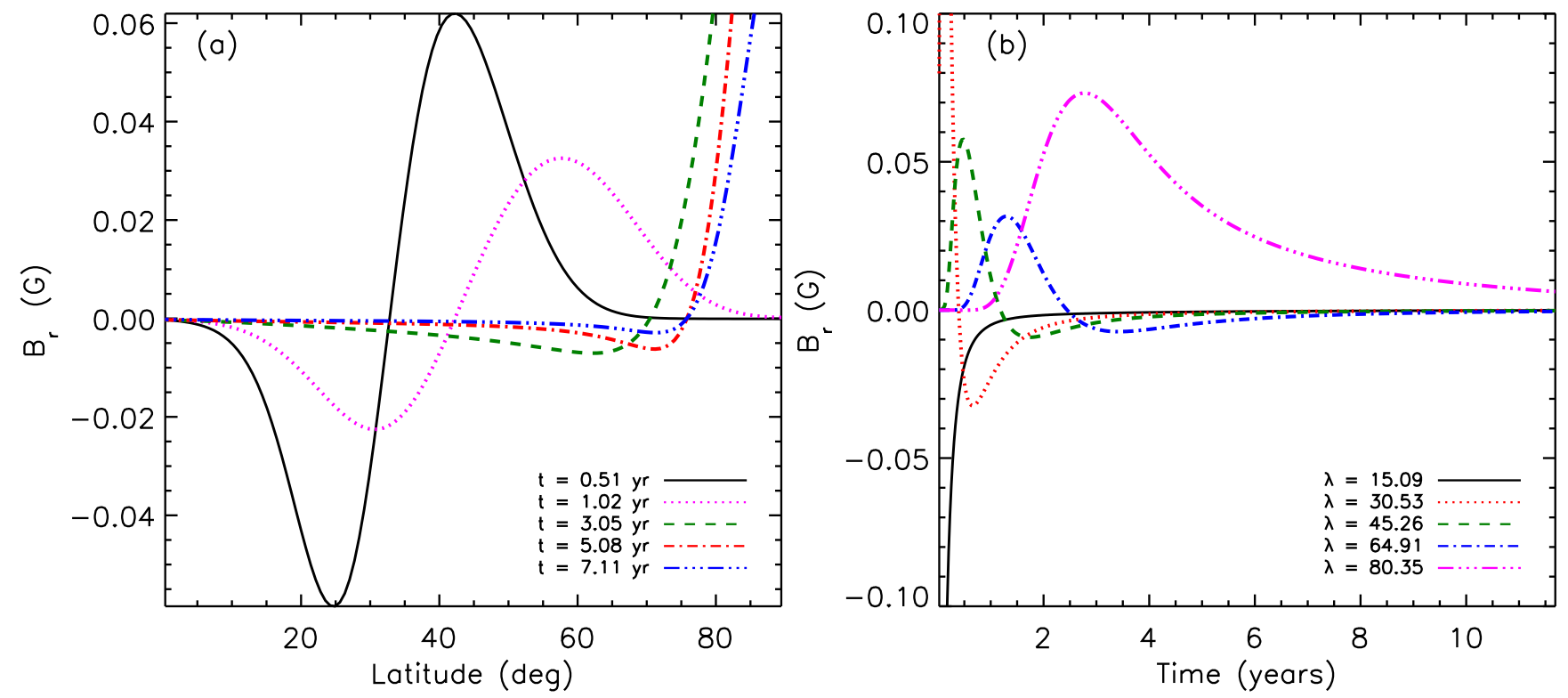

FIG. 9. - (a) Behavior of radial field with latitude is plotted for different times. Radial field just after the emergence of the sunspot (at $20^{\circ}$ latitude) are shown in black line at time $\mathrm{t}=0.51 \mathrm{yr}$. Magenta dotted, green dashed, red dash dotted, and blue long dash dotted lines represent variation of radial magnetic field with latitude at time $1.02 \mathrm{yr}, 3.05 \mathrm{yr}, 5.08 \mathrm{yr}$ and $7.11 \mathrm{yr}$ respectively. (b) Time variations of radial magnetic field for different latitudes are plotted. Solid black, red dotted, green dash dotted, blue dash dotted and magenta long dash dotted lines are for latitude $15^{\circ} .09,30^{\circ} .53,45^{\circ} .26,64^{\circ} .91$ and $80^{\circ} .35$ respectively. All units of magnetic fields are given in Gauss.
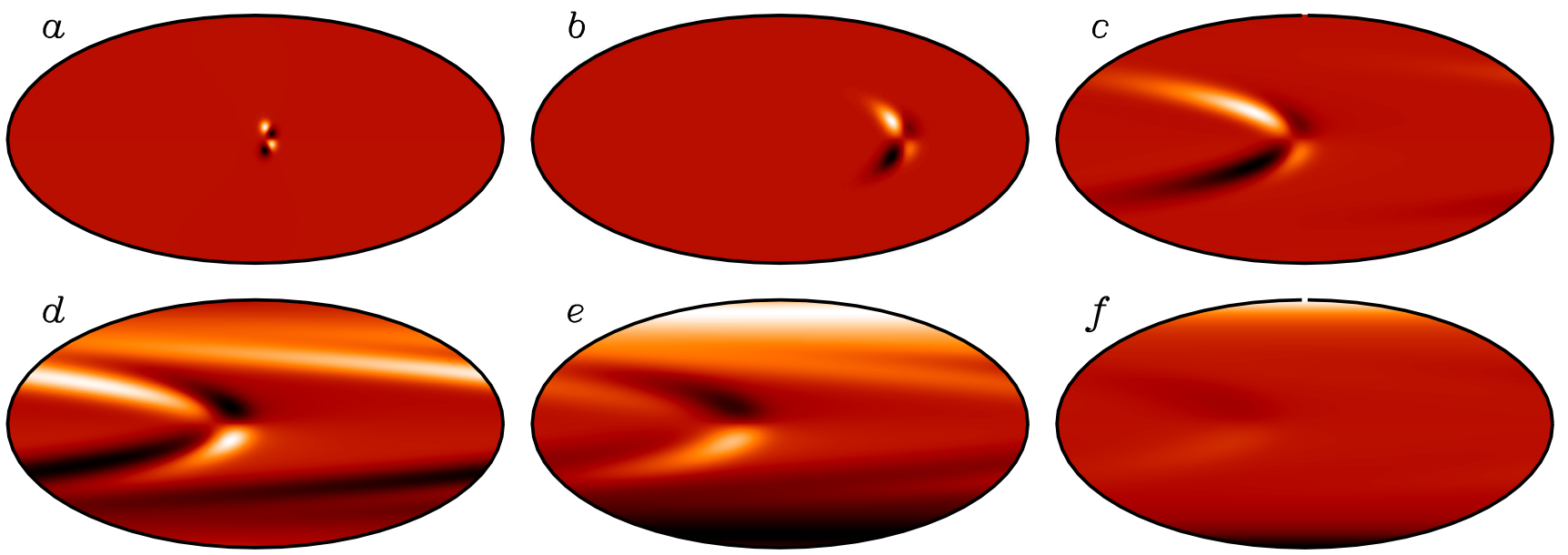

Fig. 10. - Same as Figure 7 but for sunspot emergence in two hemispheres at $\pm 5^{\circ}$ latitudes. In this figure also color scale is set at \pm maximum value of the magnetic fields for each case. 

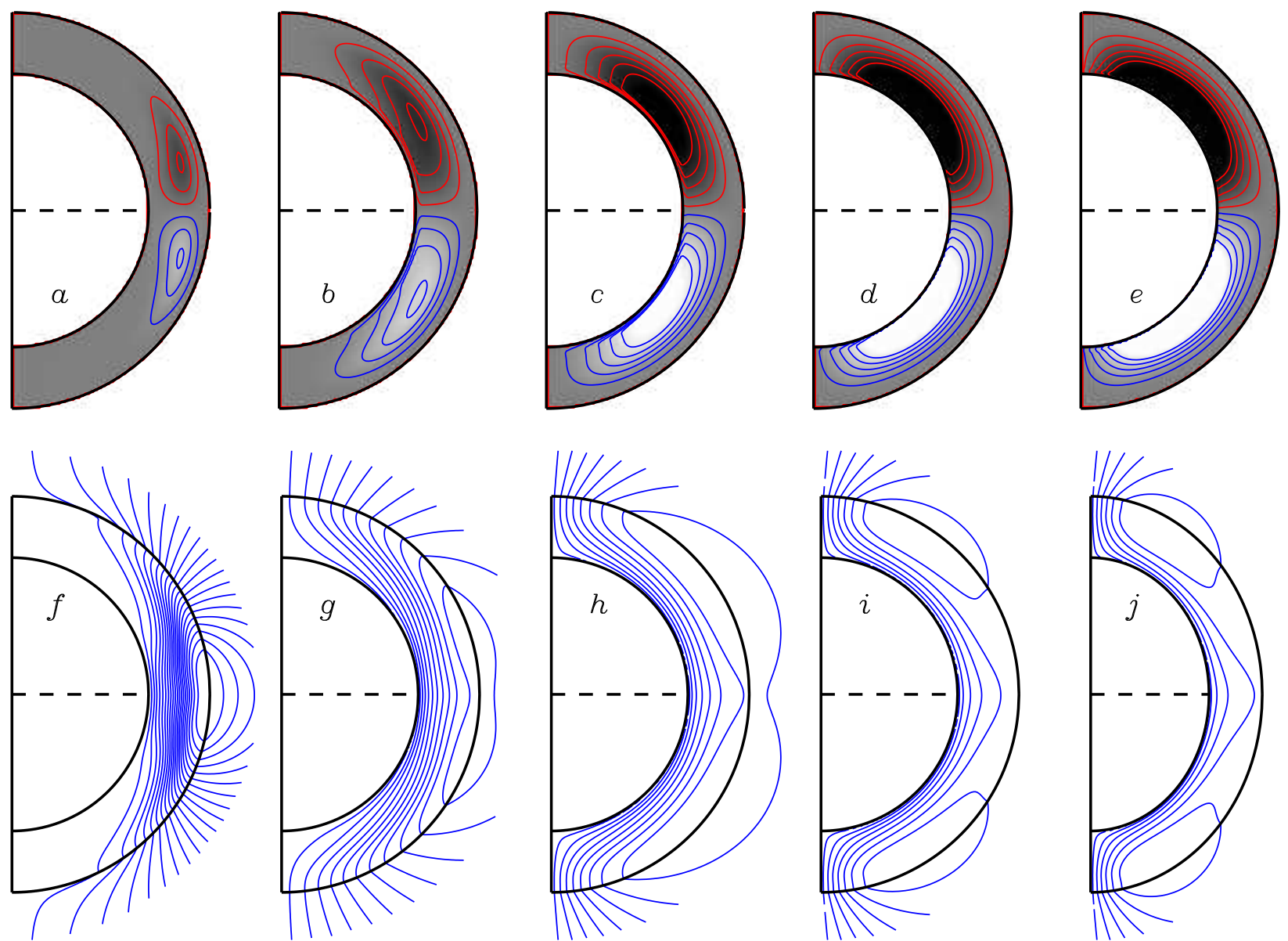

Fig. 11. - Same as Figure 8 but for two pairs at two hemispheres at $\pm 5^{\circ}$ latitudes. Color scale for toroidal fields is set at $\pm 1.5 G$ and contour levels corresponding to the poloidal fields strengths of $\pm 0.02 \mathrm{G}$ are set as maximum and minimum, respectively.
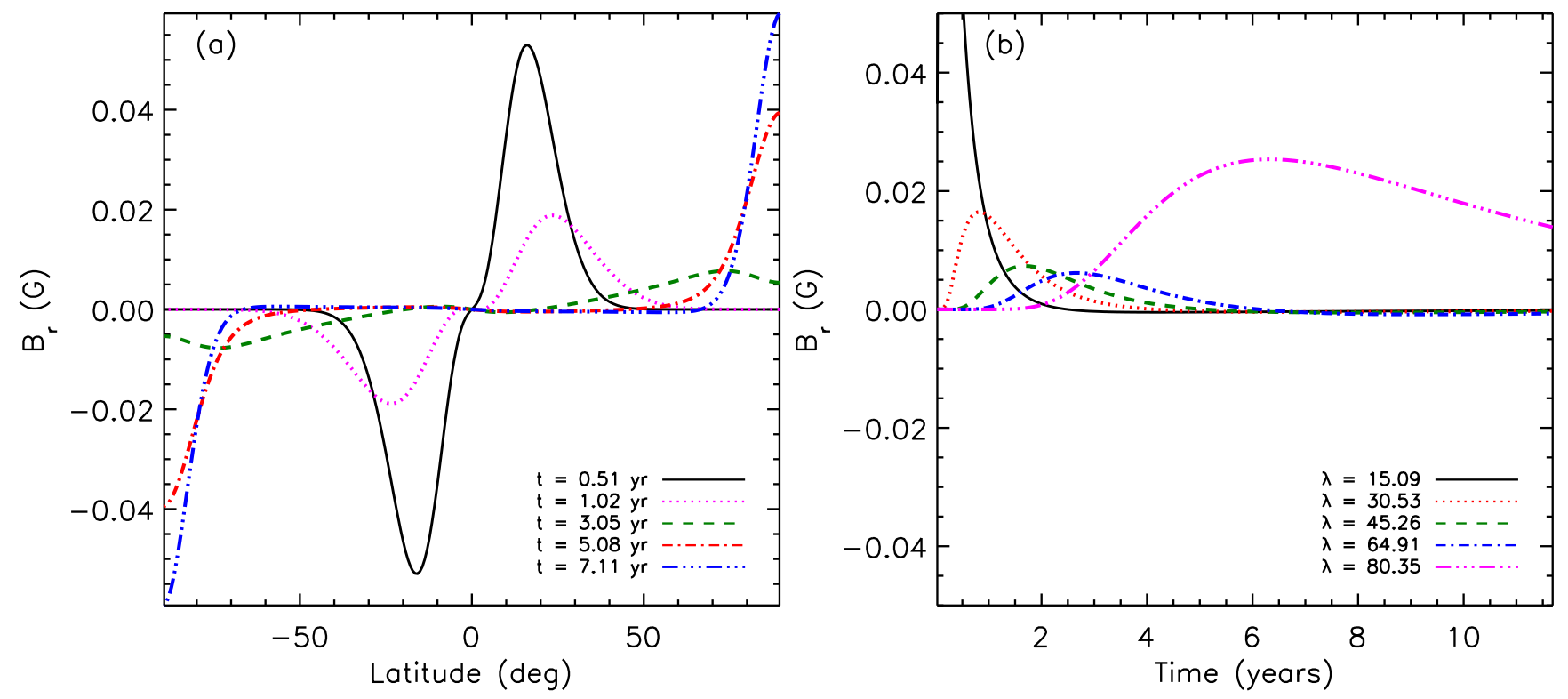

FIG. 12.- Same as Figure 9 but with two pairs in two hemispheres at $\pm 5^{\circ}$ latitudes. 
the opposite polarity of $B_{r}$ at high latitudes. This is purely a result of the $3 \mathrm{D}$ structure of the magnetic field and cannot happen in the SFT model. There would not be a source for creating $B_{r}$ at low latitudes in the SFT model and such fields would never appear in that model. Because of the breakup of the poloidal field in the two hemispheres and the appearance of $B_{r}$ with opposite polarity in the low latitudes, it is possible for the poloidal magnetic field in the 3D model to be subducted below the surface as the meridional circulation sinks downward in the polar regions. Thus, in contrast to the SFT model in which polar fields have nothing to cancel them and therefore persist, the polar field disappears after some time in the 3D model.

Though this result is notable, it may be offset to some extent by efficient magnetic pumping. Using a 2D (axisymmetric) model Karak \& Cameron (2016) have shown that downward magnetic pumping due to strongly stratified convection in the solar surface layers can suppress the upward diffusion and advection of toroidal and poloidal fields. This, in turn, can produce steady polar fields that might persist indefinitely. We will investigate the role of magnetic pumping in future work.

Figure 12(a) is similar to Figure 9(a) except that latitudes now cover from $-90^{\circ}$ to $90^{\circ}$. In this figure, we clearly see that around 1 year, we had only positive $B_{r}$ in the northern hemisphere and negative $B_{r}$ in the southern hemisphere, but afterwards very weak $B_{r}$ having sign opposite to the sign at the high latitudes developed at low latitudes. Figure 12(b), which is similar to Figure 9(b), shows that eventually $B_{r}$ disappears at the surface in this 3D model, exactly similar to what happens when we put only one sunspot pair on the solar surface.

We carry on such calculations by putting two sunspot pairs symmetrically at different latitudes in the two hemispheres. Figure 13 shows how the polar field evolves with time for sunspot pairs placed at different latitudes. When the sunspot pairs are placed at high latitudes, the magnetic flux is brought to the poles without too much diffusion and the polar field is stronger. Eventually, the polar field disappears in all the cases due to emergence and subduction by the meridional circulation, as we have already discussed. This figure can be compared with the left panel of Figure 6 of Jiang et al. (2014a). Such a comparison makes the difference between the 3D model and SFT model completely clear. In the SFT model, only if the sunspot pairs are put at sufficiently high latitudes so that cross-equatorial diffusion is negligible, fluxes of both polarity are advected to the polar regions and eventually the axial dipole moment becomes zero. If the sunspot pairs are put at low latitudes in the SFT model, only the fluxes from the following sunspots reach the poles and give rise to an asymptotic axial dipole. The situation is completely different in the 3D model, although we see that the polar field persists for a longer time when the initial sunspot pairs are put at lower latitudes. So, in that sense, sunspot pairs appearing in lower latitudes are somewhat more effective in creating the polar field even in the $3 \mathrm{D}$ model. This is in agreement with the claim of Dasi-Espuig et al. (2010) that we have a better correlation between the average tilt of a cycle and the strength of the next cycle if more weight is given to sunspot pairs at low latitudes when computing the average tilt.

We have also calculated the polar magnetic flux for two

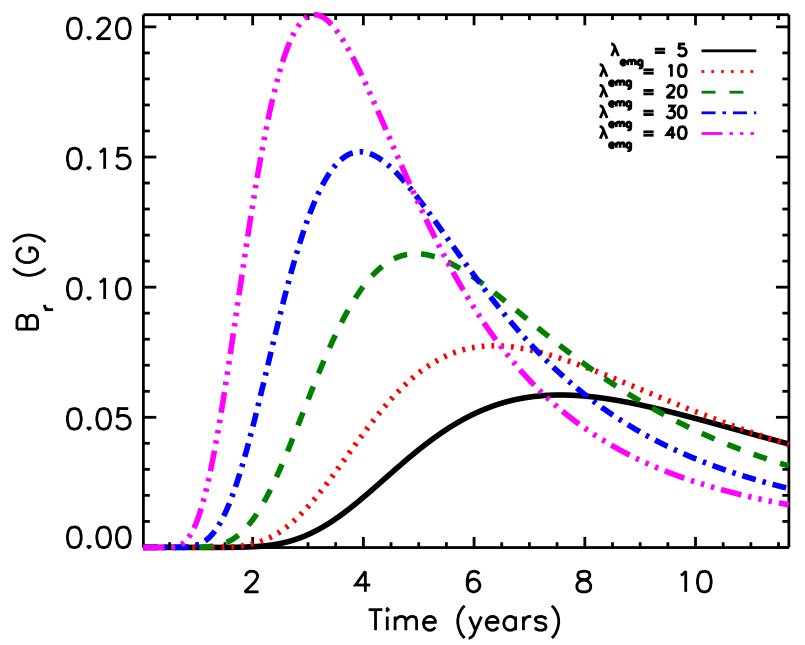

FIG. 13. - Polar field evolution with time for different emergence angle $\lambda_{\text {emg }}$ of sunspot pairs in both the hemispheres. Black solid, red dotted, green dashed, blue dash dotted and magenta long dash dotted lines represent the polar field for the sunspot emergence at $5^{\circ}, 10^{\circ}, 20^{\circ}, 30^{\circ}$, and $40^{\circ}$ respectively. Magnetic field is in Gauss and time is given in years.

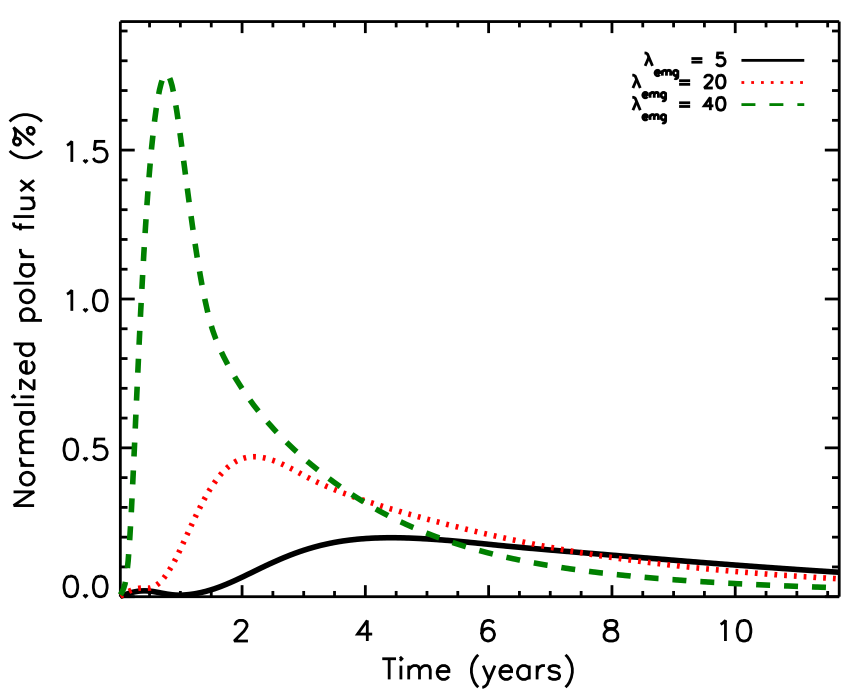

FIG. 14.- Polar flux evolution with time for different emergence angle $\lambda_{e m g}$ of sunspot pairs in both the hemisphere. Black solid, red dotted, green dashed lines represent the percentage of normalized polar flux able to reach the pole for the sunspot emergence at $5^{\circ}, 20^{\circ}$ and $40^{\circ}$ respectively.

sunspot pairs emerging on the two hemispheres, to find out how much flux from the sunspots reaches the poles. We calculate the polar flux by integrating $B_{r}$ over only those regions of the surface between $60^{\circ}$ latitude and the pole where $B_{r}$ has one sign (positive in the north pole). While positioning the sunspot pairs by hand using the SpotMaker algorithm, we injected $1 \times 10^{22} \mathrm{Mx}$ flux in each spot. A normalized polar flux is estimated by dividing the signed flux by the input flux $\left(1 \times 10^{22} \mathrm{Mx}\right)$. In Figure 14, we have shown the percentage of normalized polar flux with time for the spot pairs emerging at different latitudes. It is evident from this figure that around $1.76 \%$ of the input flux can reach the pole when the spot pair emerges at a high latitude like $40^{\circ}$, whereas $0.2 \%$ 

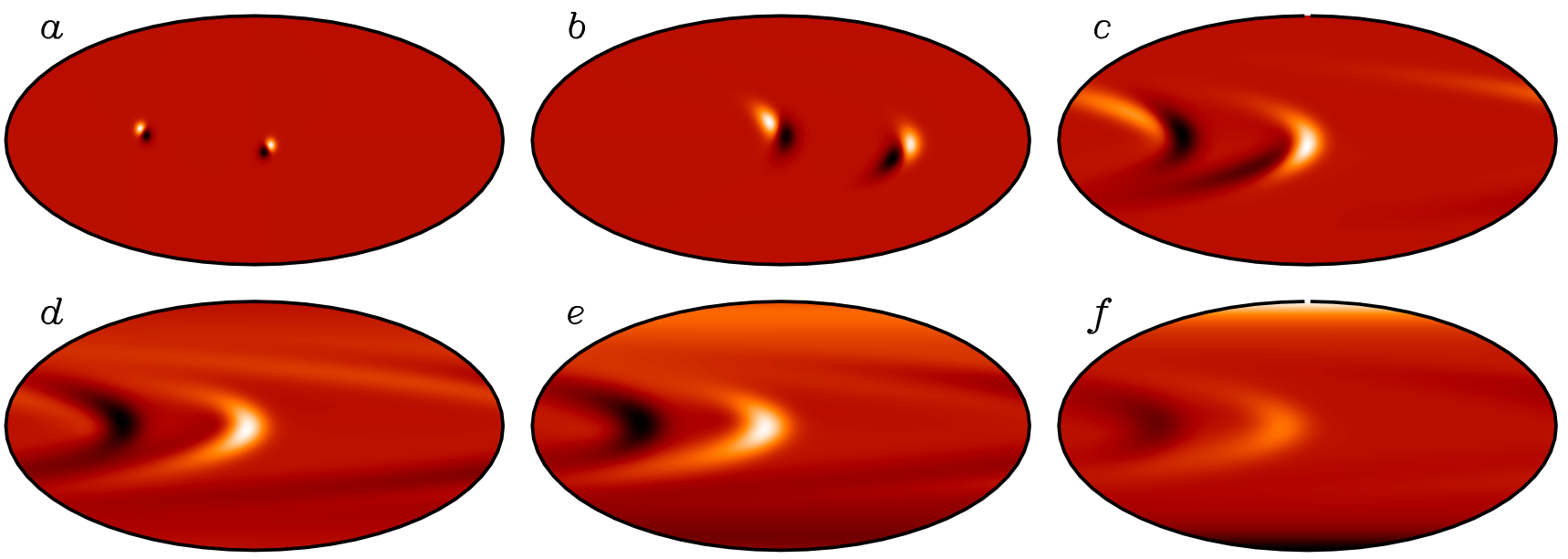

FIG. 15. - Same as Figure 10 but sunspot pairs are placed at longitude $90^{\circ}$ on northern hemisphere and at longitude $180^{\circ}$ on southern hemisphere.

of the input flux can reach the pole when the spot pair is at a low latitude like $5^{\circ}$. Keeping in mind that we have used an unrealistically high tilt of $40^{\circ}$, we point out that the flux reaching the poles will be less for more realistic tilts. It is instructive to compare our result with relevant observational data. Schrijver \& Harvey (1994) and Solanki et al. (2002) analyzed the NSO Kitt Peak magnetograph data and estimated the maximum active regions flux during solar maxima to be around $5 \times 10^{23}$ Mx. Muñoz-Jaramillo et al. (2012) calibrated century long polar faculae data from Mount Wilson Observatory and estimated the time evolution of the polar flux, finding its maximum value to be around $1.5 \times 10^{22} \mathrm{Mx}$ for an average cycle. Although these values are not from a single dataset and many other observational constraints should be taken into account, a simple division of these values of flux quoted above suggests that around $3 \%$ of the sunspots flux can contribute in the polar flux. Our theoretical model gives a value having the same order of magnitude, although our theoretical values are a little bit on the lower side.

All the results presented so far for two sunspot pairs in different hemispheres were obtained by putting both the pairs in the same longitude. This helped in magnetic fluxes of the two leading sunspots canceling each other by diffusing across the equator. One important question is whether the final outcome will be different if the two sunspot pairs in the two hemispheres are widely separated in longitude. Figure 15 shows the surface evolution of magnetic flux in such a case, which can be compared with Figure 10. We find that the magnetic fluxes from the following sunspots in the two hemispheres are carried toward the pole exactly as in Figure 10. However, the evolution of magnetic fluxes from the leading sunspots is quite intriguing. Because of the gap in longitude, these fluxes cannot cancel with each other across the equator so easily. However, these fluxes still diffuse across the equator, as seen in Figure 15, and, if we average over longitude, the averaged values are found to be virtually identical with the averaged values that we get in the case of Figure 10. When we plotted figures similar to Figure 11 and Figure 12 for this case, they turned out to be indistinguishable from Figure 11 and Figure 12.

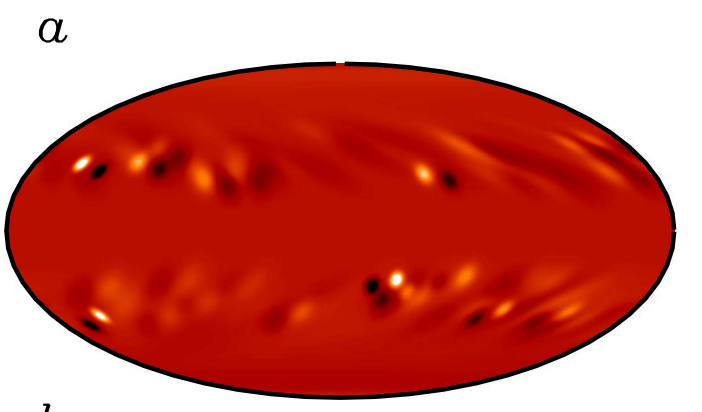

$b$

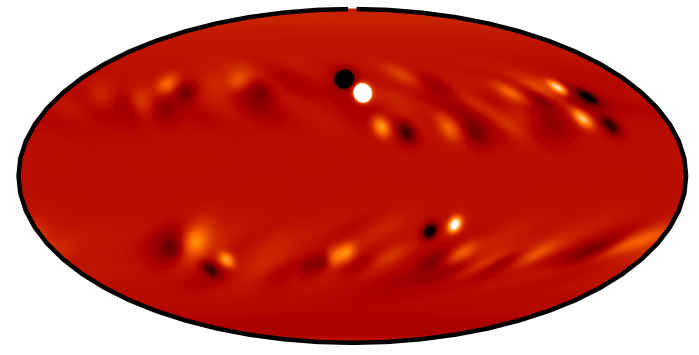

C

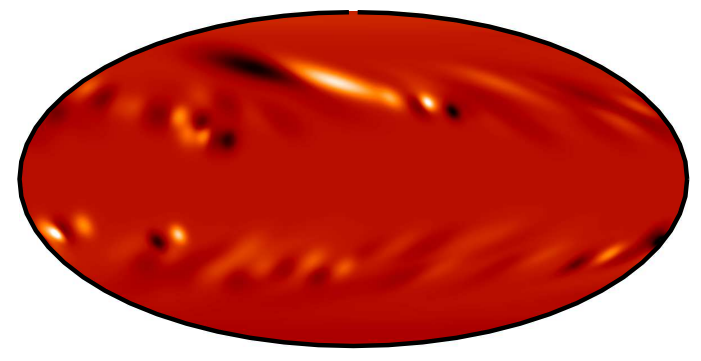

FIG. 16. - Radial magnetic field structures are shown for the case when the "anti-Hale" sunspot pair appears at $40^{\circ}$ latitude and at the middle phase of the cycle. (a) Prior to 3 months before the anti-Hale sunspot pair to be appeared, (b) During the emergence of anti-Hale sunspot pair and, (c) 3 months after the anti-Hale sunspot pair has emerged. Here white color shows the outwardgoing radial fields and black color represents inward-going radial fields. The color scale is set at $\pm 100 \mathrm{kG}$ for all three cases. 


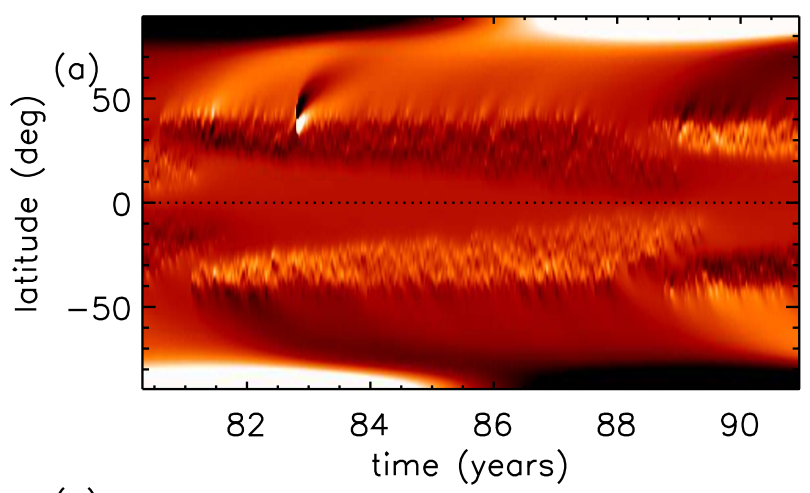

(c)

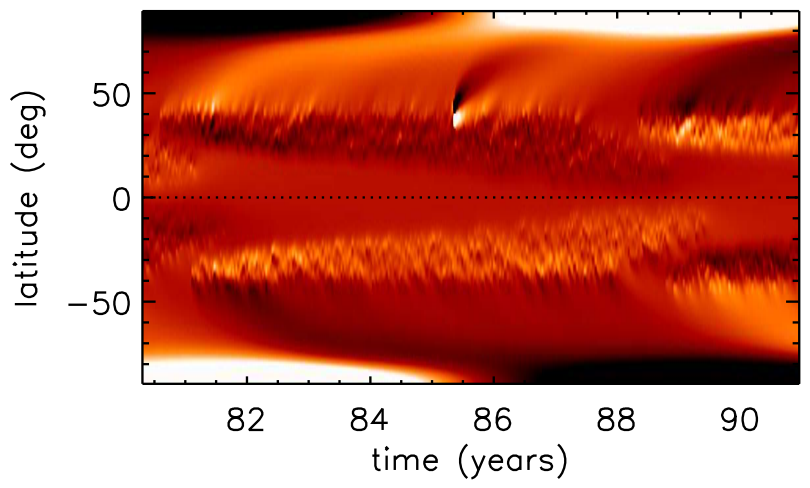

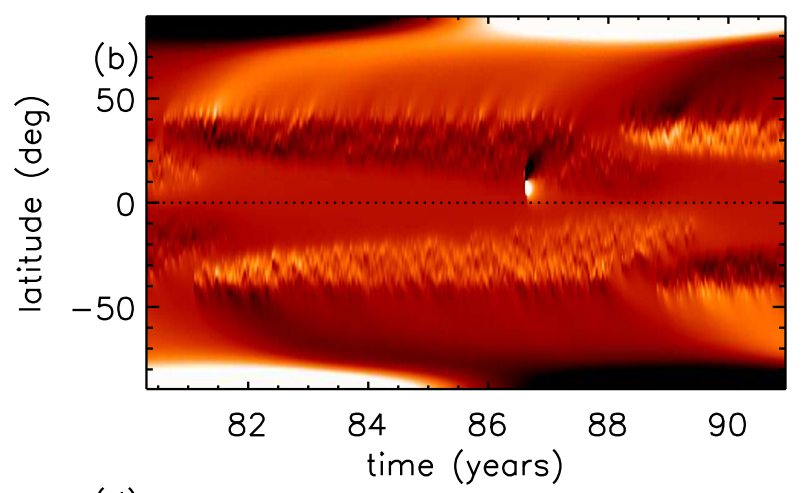

(d)

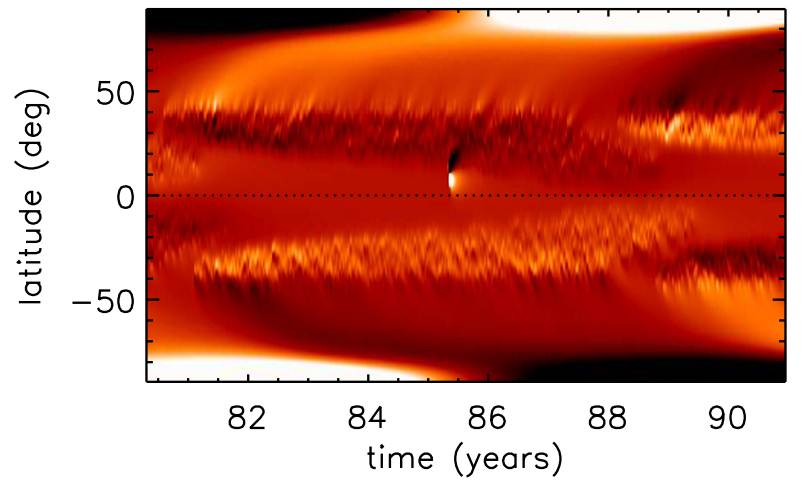

FIG. 17. - Butterfly diagram with an "anti-Hale" sunspot pair at different latitude and at different phase of the solar cycle. (a) At early phase of the cycle and at $40^{\circ}$ latitude. (b) Late phase of the cycle and at $10^{\circ}$ latitude. (c) Middle phase and at $40^{\circ}$ and (d) Middle phase and at $10^{\circ}$ latitude. Color scale is set at $\pm 15 \mathrm{kG}$ for all four cases.

\section{THE CONTRIBUTION OF BIPOLAR SUNSPOTS NOT OBEYING HALE'S LAW}

Joy's law for tilts of sunspot pairs is only a statistically average law. We see a spread of tilt angles around Joy's law. This spread is believed to be caused by the action of turbulence on rising flux tubes (Longcope \& Choudhuri 2002; Weber et al. 2011) and is one of the main sources of irregularity in the solar cycle (Choudhuri et al. 2007; Choudhuri \& Karak 2009; Choudhuri 2014). It is well known that some bipolar sunspots appear with wrong magnetic polarities not obeying Hale's polarity law. Because of the spread in tilt angles around Joy's law, it is certainly expected that a few outliers in this spread would violate Hale's law. Stenflo \& Kosovichev (2012) estimated that about $4 \%$ of medium and large sunspots violate Hale's law - see their Figure 7. Since the number of such sunspots is small, it is not surprising that due to statistical fluctuations, more of such sunspots violating Hale's law may appear in some particular cycles compared to other cycles. This fact assumes significance in the light of the suggestion made by Jiang et al. (2015) on the basis of their SFT calculations that a few large "anti-Hale" sunspot pairs may significantly decrease the strength of the polar field produced at the end of the cycle. Especially, Jiang et al. (2015) suggested that the weak polar field at the end of cycle 23 was caused by a few prominent anti-Hale sunspot pairs present in that cycle. In contrast, they argue that not too many such anti-Hale sunspot pairs appeared in cycles 21 and 22, as a result of which such a decrease of the polar field did not happen in those cycles.

Since we have seen that some insights gained from SFT calculations have to be modified-especially results connected with the build-up of the polar field - on the basis of more realistic and complete 3D kinematic dynamo calculations, we now address the question whether anti-Hale sunspot pairs have a large effect on the polar field even in 3D kinematic dynamo models. We now use our reference model presented in $\S 3$ and place a large anti-Hale sunspot pair by hand to study its effect on the build-up of the polar field. To make its effect visible, we take this anti-Hale sunspot pair to carry 25 times the magnetic flux carried by the other regular sunspots and to have tilt angle $30^{\circ}$. We can say that the tilt angle is $-30^{\circ}$, if we define the tilt angle by following the convention that its value is positive for sunspot pairs obeying Hale's law.

We want to understand how the effect of the anti-Hale sunspot pair depends on the emergence latitude, as well as the phase of the cycle, when it makes its appearance. So, we consider four different cases. Since sunspots appear at high latitudes in the early phase of the cycle and at low latitudes in the late phase, we consider one case by putting the anti-Hale sunspot pair at the high latitude of $40^{\circ}$ in the early phase and another case by putting the pair at the low latitude of $10^{\circ}$ in the late phase. The two other cases considered involve putting the large anti-Hale sunspot pair at $40^{\circ}$ and $10^{\circ}$ (in separate case studies) in the middle phase of the cycle. The radial fields on the surface in Mollweide projection is shown for a case where an "anti-Hale" sunspot pair is placed at $40^{\circ}$ latitude during the middle phase of the cycle, in Figure 16. Figure 17 shows how $B_{r}$ evolves in a time-latitude plot (a "butterfly diagram") for these four cases. The effect on the polar field can be seen more clearly in Figure 18 
where we plot the time evolution of the polar field for these four cases, along with the reference case without an anti-Hale sunspot pair.

It is clear from Figure 18 that even a very large antiHale sunspot pair placed at a low latitude like $10^{\circ}$ does not have much effect on the polar field. Presumably, the opposite fluxes from the two sunspots neutralize each other before they reach the poles. This becomes quite apparent by looking at Figures 17 (b) and $17(\mathrm{~d})$. We see that the sunspot pairs at low latitudes produce a kind of "surge" behind them, but it does not reach the poles. The effect of anti-Hale pairs at higher latitudes is certainly much more pronounced. We see in Figures 17(a) and $17(\mathrm{c})$ that the surges behind these anti-Hale pairs reach the pole in these situations. If an anti-Hale sunspot pair appears at $40^{\circ}$ in the early phase of the cycle, then we see in Figure 18 that the build-up of the polar field is weakened and delayed, but eventually the polar field reaches almost the strength we would expect in the absence of the anti-Hale sunspot pair. However, when the anti-Hale sunspot pair is put at $40^{\circ}$ in the middle phase of the cycle, it is clear from Figure 18 that polar field can be reduced by about $17 \%$. But remember that we get this large reduction by assuming the anti-Hale sunspot pair to be unrealistically large. Our conclusion is that anti-Hale sunspot pairs do affect the build-up of the polar field - especially if they appear at high latitudes in the middle phase of the cycle-but the effect does not appear to be very dramatic. As for the suggestion of Jiang et al. (2015) that the weakness of the polar field at the end of cycle 23 was due to the appearance of several anti-Hale sunspot pairs, we feel that this is an interesting suggestion which merits further detailed study in order to arrive at a firm conclusion.

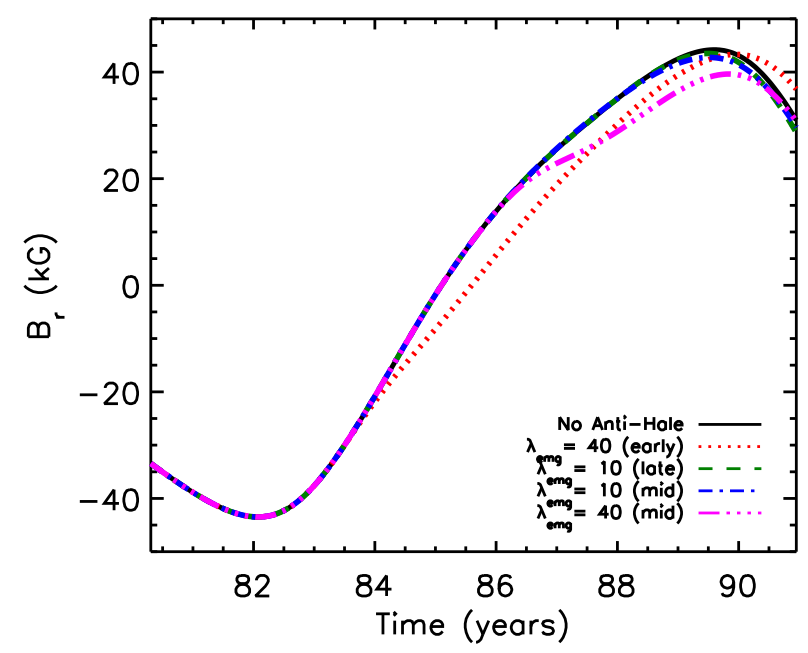

FIG. 18. - Polar field evolution with time for one complete solar cycle with the "anti-Hale" sunspot pair at different locations and different times of the cycle. Solid black line represents the regular cycle with no anti-Hale sunspot pair. Red dotted line indicates the poloidal field evolution with an anti-Hale pair at $40^{\circ}$ latitude at an early phase of the cycle. Green dashed line represents poloidal field with an anti-Hale pair at $10^{\circ}$ and late phase. Blue dashed and magenta long dashed lines indicate the poloidal field with an anti-Hale pair at middle of the cycle but at $10^{\circ}$ and at $40^{\circ}$ latitude, respectively.

\section{CONCLUSION}

Historically the evolution of the Sun's magnetic field with the solar cycle has been studied extensively through two classes of 2D theoretical models: the 2D kinematic dynamo model and the surface flux transport (SFT) model. We argue that the 3D kinematic dynamo model incorporates the attractive aspects of both, while being free from the limitations of both. On the one hand, this model can treat the Babcock-Leighton mechanism more realistically in $3 \mathrm{D}$, which is not possible in the $2 \mathrm{D}$ kinematic dynamo model. On the other hand, it includes the vectorial nature of the magnetic field and various subsurface processes which are left out in SFT models. Cameron et al. (2012) have pointed out that the results of SFT model agree with the results of 2D flux transport dynamo model on the inclusion of a downward pumping.

In order to study the build-up of the Sun's polar field with a 3D kinematic dynamo model, we first construct an appropriate self-excited model. The poloidal field generated by the Babcock-Leighton mechanism near the solar surface has to be transported to the tachocline in order for the solar dynamo to work. This transport can be achieved in two ways: (i) due to advection by the meridional circulation; or (ii) due to diffusion across the convection zone. There are reasons to believe that (ii) is the appropriate transport mechanism inside the Sun. The earlier papers by Miesch \& Dikpati (2014) and Miesch \& Teweldebirhan (2016) presented self-excited dynamo models dominated by advection by the meridional circulation. We believe that we are the first to construct self-excited 3D kinematic dynamo model dominated by diffusion. We have briefly looked at the question of parity, although the limitation of computer time prevented us from an exhaustive study of the subject.

We use this dynamo model to study how the polar field builds up from the decay of one tilted bipolar sunspot pair and two symmetrically situated bipolar sunspot pairs in the two hemispheres. We find that the polar field which arises from such sunspot pairs ultimately disappears due to the emergence of poloidal flux at low latitudes and its subsequent subduction by the meridional flow. This process is not included in the SFT models, in which the polar field can only be neutralized by diffusion with a field of opposite polarity. So we conclude that SFT models do not capture the dynamics of polar fields realistically and one has to be cautious in interpreting the SFT results pertaining to polar magnetic fields. Our results differ most dramatically from the SFT results when we put two symmetric bipolar sunspot pairs in the two hemispheres very near the equator. Then, the magnetic fields of the two leading sunspots on the two sides of the equator cancel each other. At the same time, the magnetic fields of the following sunspots which formed at higher latitudes are advected by the meridional circulation to the poles, ultimately causing a magnetic dipole of the Sun. In the SFT model, this is the whole story and we get an asymptotically steady dipole. In our 3D kinematic model, on the other hand, magnetic field lines between the two hemispheres can get detached when they are pulled by the meridional circulation in the opposite directions. As a result, radial magnetic fields with signs opposite to the polar fields develop in the lower latitudes. This is not possible in the SFT model in the absence 
of any source of radial magnetic field in the lower latitudes. Finally, the detached magnetic loops in the two hemispheres are subducted underneath the surface by the meridional circulation, contradicting the SFT result that the magnetic dipole of the Sun would be asymptotically steady in this state. While the SFT models played a tremendously important historical role in our understanding of how the magnetic field on the solar surface evolves, we should keep in mind that these models cannot capture certain aspects of the dynamics of the Sun's polar magnetic fields due the intrinsic limitations of these models.

Finally, we look into the provocative question of whether a few large sunspot pairs violating Hale's law could have a large effect on the strength of the polar field. We find that such anti-Hale sunspot pairs do pro- duce some effect on the Sun's polar field-especially if they appear at higher latitudes during the mid-phase of the solar cycle-but the effect is not very dramatic. The question of whether a few large anti-Hale sunspot pairs could be the principal cause behind the weakness of the polar field at the end of some cycles, for example cycle 23, needs to be analyzed carefully.

The computations were performed on Yellowstone cluster provided by National Center for Atmospheric Research (NCAR) and SahasraT cluster at Indian Institute of Science. We thank Bidya Binay Karak and an anonymous referee for constructive comments which helped in improving the manuscript. Partial support was provided from the JC Bose Fellowship awarded to A.R.C. by the Department of Science and Technology, Government of India. G.H. thanks CSIR, India for financial support.

\section{REFERENCES}

Babcock, H. W. 1961, ApJ, 133, 572

Baumann, I., Schmitt, D., \& Schüssler, M. 2006, A\&A, 446, 307

Baumann, I., Schmitt, D., Schüssler, M., \& Solanki, S. K. 2004, A\&A, 426, 1075

Brown, B. P., Browning, M. K., Brun, A. S., Miesch, M. S., \& Toomre, J. 2010, ApJ, 711, 424

Brun, A. S., Miesch, M. S., \& Toomre, J. 2004, ApJ, 614, 1073

Cameron, R. H., Jiang, J., Schmitt, D., \& Schüssler, M. 2010, ApJ, 719, 264

Cameron, R. H., Schmitt, D., Jiang, J., \& Işık, E. 2012, A\&A, 542, A 127

Charbonneau, P. 2014, ARA\&A, 52, 251

Chatterjee, P. \& Choudhuri, A. R. 2006, Sol. Phys., 239, 29

Chatterjee, P., Nandy, D., \& Choudhuri, A. R. 2004, A\&A, 427, 1019

Choudhuri, A. R. 1989, Sol. Phys., 123, 217

- 1998, The physics of fluids and plasmas : an introduction for astrophysicists (Cambridge: Cambridge University Press)

—. 2003, Sol. Phys., 215, 31

- 2011, Pramana, 77, 77

—. 2014, Indian Journal of Physics, 88, 877

Choudhuri, A. R., Chatterjee, P., \& Jiang, J. 2007, Physical Review Letters, 98, 131103

Choudhuri, A. R. \& Dikpati, M. 1999, Sol. Phys., 184, 61

Choudhuri, A. R. \& Hazra, G. 2016, Advances in Space Research

Choudhuri, A. R. \& Karak, B. B. 2009, Research in Astronomy and Astrophysics, 9, 953

Choudhuri, A. R., Schüssler, M., \& Dikpati, M. 1995, A\&A, 303, L29

Dasi-Espuig, M., Solanki, S. K., Krivova, N. A., Cameron, R., \& Peñuela, T. 2010, A\&A, 518, A7

Dikpati, M. \& Charbonneau, P. 1999, ApJ, 518, 508

Dikpati, M. \& Choudhuri, A. R. 1994, A\&A, 291, 975

-. 1995, Sol. Phys., 161, 9

Dikpati, M. \& Gilman, P. A. 2006, ApJ, 649, 498

D'Silva, S. \& Choudhuri, A. R. 1993, A\&A, 272, 621

Durney, B. R. 1995, Sol. Phys., 160, 213

—. 1997, ApJ, 486, 1065

Fan, Y., Fisher, G. H., \& Deluca, E. E. 1993, ApJ, 405, 390

Goel, A. \& Choudhuri, A. R. 2009, Research in Astronomy and Astrophysics, 9, 115

Guerrero, G. \& de Gouveia Dal Pino, E. M. 2007, A\&A, 464, 341 -. 2008, A\&A, 485, 267

Hale, G. E., Ellerman, F., Nicholson, S. B., \& Joy, A. H. 1919, ApJ, 49, 153

Hazra, G., Karak, B. B., \& Choudhuri, A. R. 2014, ApJ, 782, 93

Hotta, H. \& Yokoyama, T. 2010, ApJ, 714, L308

Jiang, J., Cameron, R. H., \& Schüssler, M. 2014a, ApJ, 791, 5

-. 2015, ApJ, 808, L28

Jiang, J., Chatterjee, P., \& Choudhuri, A. R. 2007, MNRAS, 381, 1527

Jiang, J., Hathaway, D. H., Cameron, R. H., Solanki, S. K., Gizon, L., \& Upton, L. 2014b, Space Sci. Rev., 186, 491

Karak, B. B. \& Cameron, R. 2016, ArXiv e-prints
Karak, B. B. \& Choudhuri, A. R. 2011, MNRAS, 410, 1503

Karak, B. B., Jiang, J., Miesch, M. S., Charbonneau, P., \&

Choudhuri, A. R. 2014, Space Sci. Rev., 186, 561

Leighton, R. B. 1964, ApJ, 140, 1547

Longcope, D. \& Choudhuri, A. R. 2002, Sol. Phys., 205, 63

Miesch, M. S. \& Dikpati, M. 2014, ApJ, 785, L8

Miesch, M. S., Elliott, J. R., Toomre, J., Clune, T. L., Glatzmaier, G. A., \& Gilman, P. A. 2000, ApJ, 532, 593

Miesch, M. S., Featherstone, N. A., Rempel, M., \& Trampedach, R. $2012,757,128(14 \mathrm{pp})$

Miesch, M. S. \& Teweldebirhan, K. 2016, Advances in Space Research

Moreno-Insertis, F. 1983, A\&A, 122, 241

Muñoz-Jaramillo, A., Nandy, D., Martens, P. C. H., \& Yeates, A. R. 2010, ApJ, 720, L20

Muñoz-Jaramillo, A., Sheeley, N. R., Zhang, J., \& DeLuca, E. E. 2012, ApJ, 753, 146

Nandy, D. \& Choudhuri, A. R. 2001, ApJ, 551, 576

Parker, E. N. 1955a, ApJ, 122, 293

-. 1955b, ApJ, 121, 491

—. 1975, ApJ, 198, 205

- 1979, Cosmical magnetic fields: Their origin and their activity (Oxford University Press)

Rajaguru, S. P. \& Antia, H. M. 2015, ApJ, 813, 114

Schad, A., Timmer, J., \& Roth, M. 2013, ApJ, 778, L38

Schou, J., Antia, H. M., Basu, S., Bogart, R. S., Bush, R. I., Chitre, S. M., Christensen-Dalsgaard, J., Di Mauro, M. P., Dziembowski, W. A., Eff-Darwich, A., Gough, D. O., Haber, D. A., Hoeksema, J. T., Howe, R., Korzennik, S. G., Kosovichev, A. G., Larsen, R. M., Pijpers, F. P., Scherrer, P. H., Sekii, T., Tarbell, T. D., Title, A. M., Thompson, M. J., \& Toomre, J. 1998, ApJ, 505, 390

Schrijver, C. J., De Rosa, M. L., \& Title, A. M. 2002, ApJ, 577, 1006

Schrijver, C. J. \& Harvey, K. L. 1994, Sol. Phys., 150, 1

Schüssler, M. \& Rempel, M. 2005, A\&A, 441, 337

Solanki, S. K., Schüssler, M., \& Fligge, M. 2002, A\&A, 383, 706

Steenbeck, M., Krause, F., \& Rädler, K.-H. 1966, Zeitschrift

Naturforschung Teil A, 21, 369

Stenflo, J. O. \& Kosovichev, A. G. 2012, ApJ, 745, 129

Thompson, M. J., Toomre, J., Anderson, E. R., Antia, H. M., Berthomieu, G., Burtonclay, D., Chitre, S. M.,

Christensen-Dalsgaard, J., Corbard, T., De Rosa, M., Genovese, C. R., Gough, D. O., Haber, D. A., Harvey, J. W., Hill, F., Howe, R., Korzennik, S. G., Kosovichev, A. G., Leibacher, J. W., Pijpers, F. P., Provost, J., Rhodes, Jr., E. J., Schou, J., Sekii, T., Stark, P. B., \& Wilson, P. R. 1996, Science, 272, 1300 Upton, L. \& Hathaway, D. H. 2014, ApJ, 780, 5

van Ballegooijen, A. A., Cartledge, N. P., \& Priest, E. R. 1998, ApJ, 501, 866

Wang, Y.-M., Nash, A. G., \& Sheeley, Jr., N. R. 1989a, ApJ, 347, 529

—. 1989b, Science, 245, 712 
Wang, Y.-M., Sheeley, Jr., N. R., \& Nash, A. G. 1991, ApJ, 383, 431

Weber, M. A., Fan, Y., \& Miesch, M. S. 2011, 741, 11 (14pp)

Yeates, A. R. \& Muñoz-Jaramillo, A. 2013, MNRAS, 436, 3366

Yeates, A. R., Nandy, D., \& Mackay, D. H. 2008, ApJ, 673, 544
Zhao, J., Bogart, R. S., Kosovichev, A. G., Duvall, Jr., T. L., \& Hartlep, T. 2013, ApJ, 774, L29 